Review Article

\title{
Comparative Efficacy of Chinese Herbal Injections Combined with Paclitaxel Plus Cisplatin for Non-Small-Cell Lung Cancer: A Multidimensional Bayesian Network Meta-Analysis
}

\author{
Mengwei Ni, ${ }^{1}$ Haojia Wang, ${ }^{1}$ Miaomiao Wang, ${ }^{1}$ Wei Zhou, ${ }^{1}$ Jiarui Wu ${ }^{1},{ }^{1}$ Bin Sun, \\ Qianqian Zhang, ${ }^{2}$ Xiaotian Fan, ${ }^{1}$ Dan Zhang, ${ }^{1}$ Zhiwei Jing, ${ }^{3}$ Jingyuan Zhang, ${ }^{1}$ Xinkui Liu, \\ Shuyu Liu, ${ }^{1}$ Ziqi Meng, ${ }^{1}$ Siyu Guo, ${ }^{1}$ Shanshan Jia, ${ }^{1}$ Xiaomeng Zhang, \\ and Xiaoguang Sheng ${ }^{1}$ \\ ${ }^{1}$ Department of Clinical Chinese Pharmacy, School of Chinese Materia Medica, Beijing University of Chinese Medicine, \\ Beijing 100102, China \\ ${ }^{2}$ Jiangsu Jiuxu Pharmaceutical Co., Ltd., Jiangsu, China \\ ${ }^{3}$ China Academy of Chinese Medicine Sciences, Beijing 100102, China \\ Correspondence should be addressed to Jiarui Wu; exogamy@163.com
}

Received 28 June 2020; Accepted 12 October 2020; Published 29 October 2020

Academic Editor: Young-Jin Son

Copyright (c) 2020 Mengwei Ni et al. This is an open access article distributed under the Creative Commons Attribution License, which permits unrestricted use, distribution, and reproduction in any medium, provided the original work is properly cited.

\begin{abstract}
Introduction. Considering the limitations of pure paclitaxel plus cisplatin chemotherapy in the treatment of non-small-cell lung cancer and the extensive exploration of Chinese herbal injections, this study performed a multidimensional network meta-analysis to systematically evaluate the clinical efficacy and safety of 12 Chinese herbal injections in the treatment of non-small-cell lung cancer. Methods. Randomized controlled trials were obtained from several databases according to the eligibility criteria, and the study quality was assessed by the Cochrane risk of bias tool. Data analysis was performed by Stata 13.1 software and WinBUGS 14.0 software. Multidimensional cluster analysis was performed with the "scatterplot3d" package in $R$ 3.6.1 software (PROSPERO ID: CRD42020163503). Results. A total of 58 eligible randomized controlled trials involving 4578 patients and 12 Chinese herbal injections were included. Combined with paclitaxel plus cisplatin chemotherapy, Xiaoaiping injection exhibited a better impact on the clinical effective rate than paclitaxel plus cisplatin alone. Shenqifuzheng injection was associated with a preferable response in performance status and reduced leukopenia and gastrointestinal reactions. Kangai injection was dominant in the comprehensive results of the cluster analysis. Conclusions. Chinese herbal injections combined with paclitaxel plus cisplatin chemotherapy have a certain adjuvant effect in treating non-small-cell lung cancer, but the results of this study need to be verified by more welldesigned, large-sample, multicenter randomized controlled trials.
\end{abstract}

\section{Introduction}

According to the latest global cancer report, both the incidence and mortality of lung cancer ranked first among malignant tumors. Currently, the survival rate of most cancers is steadily increasing, while the 5-year relative survival rate of lung cancer is still stagnant, at 18\% [1-4]. Primary bronchial lung cancer (referred to as lung cancer) is internationally divided into small-cell lung cancer and nonsmall-cell lung cancer (NSCLC) [5]. NSCLC accounts for approximately $85 \%$ of the total number of lung cancer cases. This value has still been increasing in the recent years, and more than $75 \%$ of patients are already in an advanced stage of lung cancer at the time of initial diagnosis and miss the opportunity for surgical treatment [6-8]. In the recent years, the treatment of NSCLC has improved, and its primary therapeutic regimens involve the combination of two drugs based on platinum drugs, such as vinorelbine plus cisplatin, paclitaxel plus cisplatin (TP), and gemcitabine plus cisplatin [9-11]. TP is a commonly used drug in chemotherapy for 
patients with NSCLC, and its antitumor efficacy has been demonstrated [12-16]. However, TP chemotherapy is often accompanied by the occurrence of adverse events, and how to improve clinical efficacy and reduce adverse reactions has attracted much attention $[17,18]$.

In China, the treatment of traditional Chinese medicine combined with chemotherapy for cancer has been widely used $[19,20]$. Studies have shown that traditional Chinese medicine has beneficial effects on treating cancer, delaying cancer progression, reducing the complications caused by chemotherapy, and reducing adverse events [21-23]. Chinese herbal injections (CHIs), as an integral part of traditional Chinese medicine, play a vital role in treating cancer. Studies have shown that many components in Xiaoaiping injection have significant antitumor effects. Among them, there are dozens of C-21 steroidal saponins, which are the main anticancer components [24]. Modern pharmacological studies have indicated that these components can inhibit tumor cell proliferation, induce apoptosis, reduce the drug resistance of antitumor drugs, and improve body immunity [25-30].

Network meta-analysis (NMA) was developed from traditional meta-analysis, extending from the standard twoarm meta-analysis to indirect comparisons of multiple related but different interventions at the same time [31]. While CHIs are widely used in clinical practice, the comparison of the efficacy between multiple varieties cannot be achieved through traditional meta-analysis methods. The greatest advantage of the NMA method is that it can summarize different interventions for the treatment of a certain kind of disease, then carry out quantitative statistical analysis and rank these interventions according to the results of one outcome indicator, and finally, choose the optimal treatment plan. This NMA compares the efficacy of 12 CHIs combined with TP chemotherapy in the treatment of NSCLC by quantitatively synthesizing the evidence. This study aims to provide a reference for the clinical application of $\mathrm{CHI}$ to assist in the treatment of NSCLC.

\section{Method}

This NMA was conducted according to the Preferred Reporting Items for Systematic Reviews and Meta-Analyses (PRISMA) extension statement for reporting of systematic reviews incorporating network meta-analyses of healthcare interventions [32]. A completed PRISMA checklist is included as an additional file (Checklist). A protocol, including the full methods for this review, is available from https://www.crd.york.ac.uk/prospero/ display_record.php?ID=CRD42020163503.

2.1. Inclusion and Exclusion Criteria. Studies were considered eligible for inclusion if the following criteria were met. (1) Randomized controlled trials (RCTs) of CHIs combined with the TP chemotherapy regimen for treating NSCLC. Studies mentioning "random" were included, regardless of whether blinding methods were used. (2) All patients were diagnosed with stage III or stage IV NSCLC and had an exact pathological or cytological diagnosis. Sex and race were not limited. (3) The control group was treated with the TP chemotherapy regimen, while the observation group received CHI by intravenous drip and the TP chemotherapy regimen. There was no limitation on the dosages or treatment courses, but other adjuvant therapies (such as Chinese herbal decoction, other Chinese patent medicines, and surgical treatment) should not be used. Symptomatic treatments were given if other complications occurred. (4) The study described efficacy outcomes, such as the clinical effective rate, performance status, leukopenia, and gastrointestinal reactions $[33,34]$. The primary outcome was the clinical effective rate, according to WHO's criteria for evaluating the efficacy of solid tumors, which could be divided into four levels: complete response (CR), in which visible lesions completely disappeared after $>1$ month; partial response (PR), in which the tumor area of a single lesion was reduced by $\geq 50 \%$ or the sum of the two largest vertical diameter products of multiple lesions was reduced by $>50 \%$; stable disease (SD), in which no significant change in condition occurred for, at least, 4 weeks and the tumor size was estimated to increase by $<25 \%$ or decrease by $<50 \%$; and progressive disease (PD), in which new lesions appeared or original lesions were estimated to increase by $\geq 25 \%$. The clinical effective rate was calculated by the following formula: clinical effective rate $=$ (number of $\mathrm{CR}$ patient$s+$ number of PR patients)/total number of patients $\times 100 \%$. The secondary outcomes of interest were performance status and adverse drug reactions/adverse drug events (ADRs/ ADEs), including leukopenia and gastrointestinal reactions. The performance status was assessed by the Karnofsky performance status (KPS) score. KPS scores that increased by $\geq 10$ points after treatment were considered to improve the performance status; KPS scores that decreased by $\geq 10$ points after treatment were considered to reduce the performance status; and KPS scores that increased or decreased by $<10$ points were considered stable. The performance status improvement rate $=$ number of patients with improved performance status/total number of patients $\times 100 \%$. Referring to the "Acute and Subacute Toxicity Standards of Chemotherapy Drugs" formulated by the WHO in 1981, ADRs/ADEs were divided into 5 grades. The incidence of $\mathrm{ADRs} / \mathrm{ADEs}=$ number of patients with ADRs/total number of patients $\times 100 \%$.

RCTs were excluded if they met the following criteria: (1) patients with other primary tumors; (2) the intervention measures were non-TP chemotherapy, or the intervention was combined with other Chinese medical treatments (such as Chinese medicine prescriptions, Chinese patent medicines, or massage), as well as Western medical treatments such as radiotherapy and surgery; (3) the administration of CHIs was nonintravenous; (4) nonclinical trials, self-controlled studies, or studies using incorrect randomization methods; (5) duplicate studies (only those with the most recent publication year, larger sample size, and more comprehensive information were retained); (6) studies with incomplete or erroneous data such as the drug name, dosage, and course of treatment; and (7) studies with inconsistent evaluation criteria and those with no related outcome indicators. 
2.2. Search Strategy. In this NMA, RCTs were retrieved from the inception of the database to November 30, 2019, from the following databases: Embase, PubMed, the Cochrane Library, the China National Knowledge Infrastructure Database (CNKI), the Wan-fang Database, the CQVIP Database (VIP), and the China Biology Medicine disc (SinoMed). Unpublished studies and related academic organizations' websites were also researched to supplement RCTs of CHIs combined with chemotherapy for NSCLC. The search strategy, using a combination of mesh terms and free text search terms, included three parts: NSCLC, CHIs, and RCTs. Using PubMed as an example, "Non-Small-Cell Lung Carcinomas [MeSH Terms]," "Non-Small-Cell Lung Carcinoma," "Non-Small-Cell Lung Cancer," "Non-Small-Cell Lung Carcinoma," "Non-Small-Cell Lung Carcinoma," and "Non-Small-Cell Lung Cancer" were applied to identify relevant publications on NSCLC. More details about the strategy of CHIs are provided in Supplementary Materials (available here).

2.3. Study Selection and Data Extraction. Two researchers independently screened the titles and abstracts of potential articles to remove duplicate studies and exclude studies that definitely did not meet the inclusion criteria, and then, they cross checked the results of the included studies. Regarding repeated reports, for example, a report on the results of different periods of the same trial was included as only 1 study. Any divergences were resolved by discussion or the third reviewer. The main components of the extracted data were as follows: (1) basic information of included studies, including the title, first author's name, publication year, and source of the literature; (2) characteristics of patients, including number of patients, age, sex, KPS score before treatment, type of tumor, and clinical stage; (3) interventions, including name, dosage, course of treatment, and treatment cycle of CHIs, as well as chemotherapy drugs; (4) outcome indicators: measured data on the clinical effective rate, performance status, and ADRs/ADEs (leukopenia and gastrointestinal reactions); and (5) quality assessment items, including key factors for the type of study design and risk of bias assessment.

2.4. Risk of Bias Assessment. The Cochrane risk of bias tool (Cochrane Handbook for Systematic Reviews of Interventions, version 5.1.0) [35] was used to evaluate the quality of the eligible RCTs by 2 researchers. Any divergences were resolved by discussion or the third reviewer. Based on the characteristics of CHIs, we formulated quality evaluation items for the RCTs as follows: (1) whether random sequence generation and allocation concealment were mentioned (selection bias) and whether follow-up information was mentioned, including the number and reasons for withdrawals and lost follow-ups; (2) whether the blinding of the participants and personnel was mentioned (performance bias); (3) whether the blinding of the outcome assessment was mentioned (detection bias); (4) whether incomplete outcome data were mentioned (attrition bias); (5) description of eligibility criteria; (6) evaluation of ADRs/ADEs;
(7) description of the statistical method; (8) funding; and (9) medical ethics.

2.5. Statistical Analysis. First, heterogeneity analysis was performed using the chi-square test, and the amount of heterogeneity was evaluated with the $I^{2}$ statistic. It is suitable to perform NMA when slight heterogeneity exists $\left(I^{2} \leq 50 \%\right)$. However, if substantial heterogeneity exists $\left(I^{2}>50 \%\right)$, the causes of heterogeneity should first be considered. Then, we decided whether to use a random effects model after excluding clinical heterogeneity factors and, finally, performed a combined analysis. No consistency test was needed in this study because it was based on an indirect comparison comparing different varieties of CHIs combined with chemotherapy. An NMA evidence relationship diagram was performed by Stata 13.1 software (Stata Corp, College Station, TX, USA), which presents indirect comparative relationships between different interventions [36]. The statistical analysis was performed by WinBUGS 14.0 software (MRC Biostatistics Unit, Cambridge, UK). Advanced Bayesian inference with the Markov Chain Monte Carlo (MCMC) method was performed, in which the posterior probability was inferred from the prior probability and, then, estimated and extrapolated under the assumption that MCMC has reached a stable convergence state. When running the WinBUGS program, the number of iterations was set to 200 000, of which the first 10000 iterations were used for annealing and to eliminate the effect of the initial value. The results of the indirect comparisons are expressed as the median and $95 \%$ confidence interval (95\% CI) of the odds ratio (OR), which was used as the effect statistic. The relative ordering of effectiveness was obtained according to the ranking result of the median ORs. According to the surface under the cumulative ranking curve (SUCRA) value, which ranged from 0 to 1 , the ranking probabilities of different interventions could be estimated; the intervention is absolutely effective when the SUCRA value is 1 , while the intervention is absolutely ineffective when it is 0 [37-40]. Then, based on the SUCRA value, clustering analysis was performed on the outcome indicators, aiming to obtain the best intervention among the two clustering indicators [41]. A symmetrical comparison-adjusted funnel plot was used to assess publication bias and showed that there was no obvious publication bias in this study $[42,43]$.

2.6. Multidimensional Cluster Analysis. Multidimensional cluster analysis of the outcome indicators was performed with the "scatterplot3d" package in $R$ 3.6.1 software (Mathsoft, Cambridge, USA). Using the $k$-means method to cluster these interventions, the number of clusters was adjusted according to the actual problem. The steps of $k$ means clustering were as follows: (1) All interventions were randomly divided into $k$ initial classes, and the average of the outcome indicators of these $k$ classes was used as the initial aggregation point. (2) Each intervention was classified into the category of the closest aggregation point; then, the aggregation point of this category was updated to the average of the current outcome indicators. All interventions were 
recategorized and classified. Step (2) was repeated until all interventions had been assigned, and finally, the ranking of the interventions with three outcome indicators was visualized through a three-dimensional stereogram. Different colors were applied to indicate interventions belonging to different categories.

\section{Results}

3.1. Literature Selection. A total of 6593 articles were retrieved in accordance with the predetermined search strategy and data collection method. After deduplication, reading the titles and abstracts, and excluding significantly the irrelevant literature, a total of 1122 RCTs of CHIs combined with the TP chemotherapy regimen in the treatment of NSCLC were collected. After reviewing the full texts of these studies, 58 RCTs [44-64], including [65-90] 12 kinds [91-101] of CHIs (Aidi injection, Huangqiduotang injection, compound Kushen injection, Delisheng injection, Huanchansu injection, Yadanziyouru injection, Kangai injection, Kanglaite injection, Shenfu injection, Shenmai injection, Shenqifuzheng injection, and Xiaoaiping injection) were included in this study. All of the studies included were Chinese literature. The study identification, screening, and inclusion process is illustrated in Figure 1.

3.2. Study Characteristics. A total of 4578 patients were included in this study, with 2310 patients in the observation group and 2268 in the control group. All included RCTs reported the number of patients, as well as their sex, age, TNM stage, estimated survival, and KPS score before treatment. The TP chemotherapy regimen was given to the control group, and the observation group received $\mathrm{CHI}$ intravenously. Information on the types, doses, and treatment courses of CHIs in the observation group and information on the doses of chemotherapeutic drugs in the control group is provided in Table 1. Figure 2 shows the network relationship of CHIs combined with TP chemotherapy in the treatment of NSCLC.

3.3. Quality Evaluation. Thirteen RCTs (22.41\%) generated random sequences with the random number table method, and 1 RCT $(1.72 \%)$ used the lottery method, so their selection bias due to random sequence generation was evaluated as "low risk." The remaining RCTs that only mentioned "random" were evaluated as "unclear." Two RCTs (3.45\%) mentioned the implementation of a blinding method, and 1 RCT (1.72\%) mentioned the use of a hidden random sequence, so their performance bias and detection bias were evaluated as "low risk." Most RCTs that did not apply random sequence concealment and blindness were evaluated as "unclear." In addition, as all included studies did not have incomplete outcomes or selective reporting, their attrition bias, reporting bias, and other bias were all evaluated as "low risk." Specific information about the risk of bias is shown in Figure 3.
3.4. Clinical Effective Rate. The NMA on the clinical effective rate included $50 \mathrm{RCTs}, 12$ interventions, and 11 kinds of CHIs. The following CHIs combined with TP chemotherapy were significantly more effective than using TP chemotherapy alone: Aidi injection $(\mathrm{OR}=0.64 ; 95 \% \mathrm{CI}, 0.47-0.86)$, compound Kushen injection $(\mathrm{OR}=0.54 ; 95 \% \mathrm{CI}, 0.37-0.77)$, Kangai injection $(\mathrm{OR}=0.45 ; 95 \% \mathrm{CI}, 0.30-0.66)$, Kanglaite injection $(\mathrm{OR}=0.49 ; 95 \% \mathrm{CI}, 0.32-0.76)$, Shenmai injection $(\mathrm{OR}=0.86 ; 95 \% \mathrm{CI}, 0.29-2.46)$, and Yadanziyouru injection (OR $=0.39 ; 95 \% \mathrm{CI}, 0.23-0.64)$. Based on TP chemotherapy, Xiaoaiping injection might hold greater potential for increasing the clinical effective rate than Delisheng injection $(\mathrm{OR}=2.18$; 95\% CI, 1.05-4.58), and the difference between the groups was statistically significant. There were no significant differences in the clinical effective rate between the other CHIs (Table 2).

Based on the ranking results of improving the clinical effective rate, the relative ranking of interventions was as follows: Xiaoaiping injection $(88.2 \%)>$ Kangai injection $(79.2 \%)>$ Huachansu injection $(73.95 \%)>$ Kanglaite injection $(71.62 \%)>$ compound Kushen injection (64.13\%) > Aidi injection (47.07\%) > Yadanziyouru injection (39.35\%) $>$ Shenqifuzheng injection $(38.13 \%)>$ Shenmai injection $(31.66 \%)>$ Huangqiduotang injection $(29.72 \%)>$ Delisheng injection (25.98\%) > TP only (10.99\%) (Figure 4).

3.5. Performance Status. The NMA for performance status included 41 RCTs, 11 interventions, and 10 kinds of CHIs. The following CHIs combined with TP chemotherapy were significantly more effective in improving performance status than using TP chemotherapy alone: Aidi injection $(\mathrm{OR}=0.37 ; 95 \% \mathrm{CI}, 0.25-0.53)$, compound Kushen injection $(\mathrm{OR}=0.30 ; 95 \%$ CI, 0.17-0.49), Huachansu injection $(\mathrm{OR}=0.31 ; 95 \% \mathrm{CI}, 0.15-0.67)$, Yadanziyouru injection $(\mathrm{OR}=0.27 ; 95 \% \mathrm{CI}, 0.15-0.48)$, Kangai injection $(\mathrm{OR}=0.30$; 95\% CI, 0.19-0.47), Kanglaite injection $(\mathrm{OR}=0.29 ; 95 \% \mathrm{CI}$, 0.11-0.73), Shenqifuzheng injection $(\mathrm{OR}=0.22 ; 95 \% \mathrm{CI}$, $0.15-0.34)$, and Xiaoaiping injection $(\mathrm{OR}=0.38$; $95 \% \mathrm{CI}$, 0.19-0.74) (Table 2).

Based on the ranking results of improving performance status, the relative ranking of interventions was as follows: Shenqifuzheng injection $(81.82 \%)>$ Yadanziyouru injection $(64.72 \%)>$ compound Kushen injection (58.32\%) $>$ Kanglaite injection $(57.79 \%)>$ Shenmai injection $(56.61 \%)>$ Kangai injection $(55.99 \%)>$ Huachansu injection $(52.59 \%)>$ Delisheng injection $(47.58 \%)>$ Xiaoaiping injection (37.54\%) $>$ Aidi injection (36.61) $>$ TP only $(0.44 \%)$ (Figure 4).

\subsection{ADRs/ADEs}

3.6.1. Leukopenia. The NMA for leukopenia included 31 RCTs, 11 interventions, and 10 kinds of CHIs. The following CHIs combined with TP chemotherapy were significantly more effective in reducing leukopenia than TP chemotherapy alone: compound Kushen injection $(\mathrm{OR}=2.72 ; 95 \%$ CI, 1.17-6.63), Yadanziyouru injection ( $\mathrm{OR}=3.10 ; 95 \% \mathrm{CI}$, 1.09-9.06), Kangai injection $(\mathrm{OR}=6.30 ; 95 \% \quad \mathrm{CI}$, 


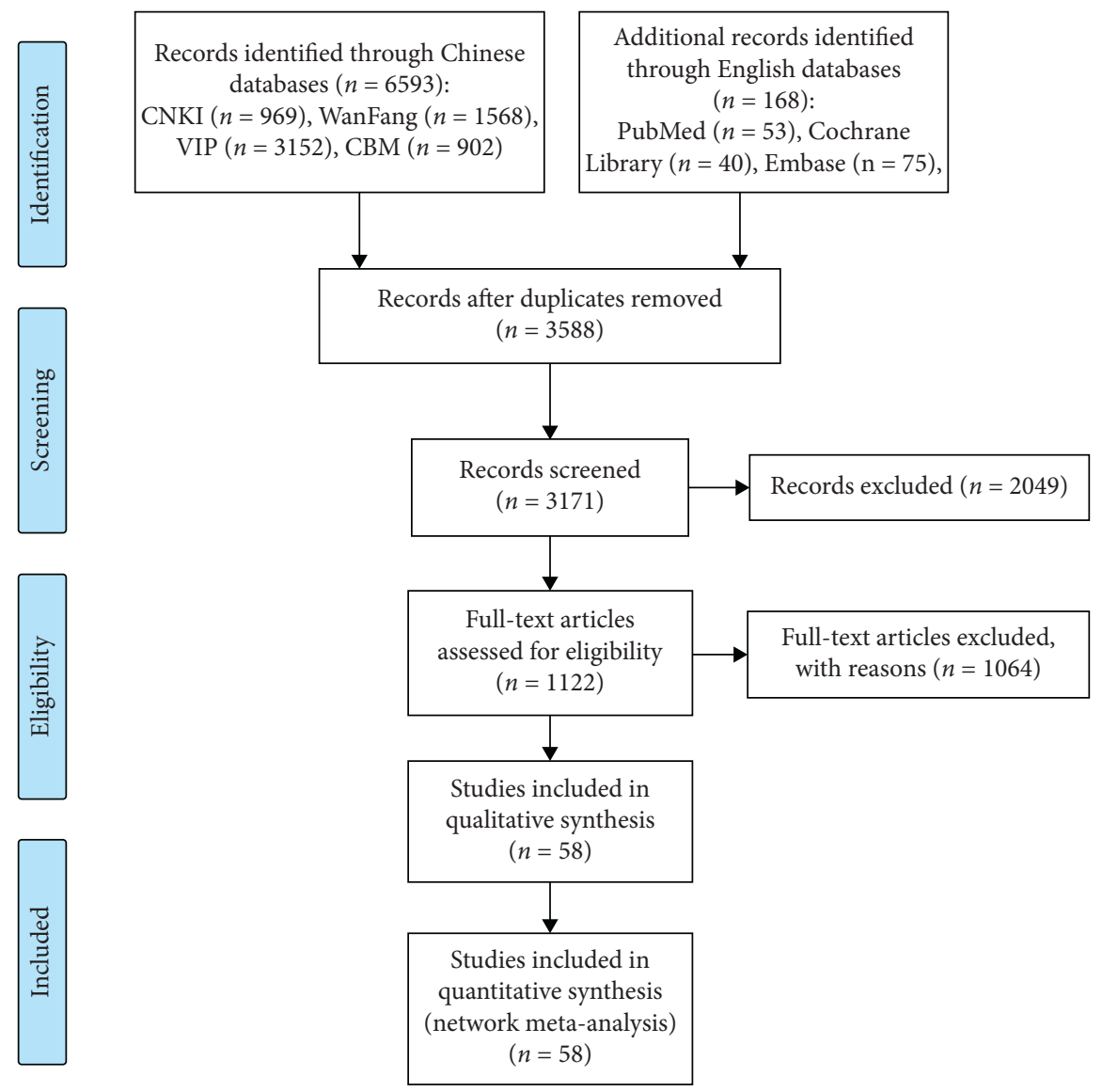

FIGURE 1: PRISMA flow diagram. (n, number of articles; CNKI, China National Knowledge Infrastructure Database; Wan-Fang, the WanFang Database; VIP, the Chinese Scientific Journals Full-Text Database; and CBM, the Chinese Biomedical Literature Database).

1.77-23.12), and Shenqifuzheng injection ( $\mathrm{OR}=5.81 ; 95 \%$ CI, 3.06-11.29). Based on TP chemotherapy, Shenqifuzheng injection might hold greater potential for reducing leukopenia than Aidi injection $(\mathrm{OR}=0.34 ; 95 \% \mathrm{CI}, 0.13-0.92)$, and the difference between the groups was statistically significant. There were no significant differences in leukopenia between the other CHIs (Table 3).

Based on the ranking results of reducing leukopenia, the relative ranking of interventions was as follows: Shenqifuzheng injection (82.63\%) $>$ Kangai injection $(81.24 \%)$ $>$ Shenfu injection $(64.46 \%)>$ Shenmai injection $(56.24 \%)$ $>$ Yadanziyouru injection (54.25\%) > Delisheng injection $(51.07 \%)>$ compound Kushen injection (48.32\%) $(52.59 \%)$ $>$ Kanglaite injection (39.05\%) $>$ Xiaoaiping injection $(34.74 \%)>$ Aidi injection $(33.14 \%)>$ TP only $(4.851 \%)$ (Figure 4).

3.6.2. Gastrointestinal Reactions. The NMA for gastrointestinal reactions included 39 RCTs, 12 interventions, and 11 kinds of CHIs. The following CHIs combined with TP chemotherapy were significantly more effective in reducing gastrointestinal reactions than TP chemotherapy alone: Kangai injection $(\mathrm{OR}=3.32 ; 95 \% \mathrm{CI}, 1.51-7.38)$, and Shenqifuzheng injection ( $\mathrm{OR}=8.20 ; 95 \% \mathrm{CI}, 3.83-18.39)$. Based on TP chemotherapy, Shenqifuzheng injection might hold greater potential for reducing gastrointestinal reactions than Aidi injection ( $\mathrm{OR}=0.19 ; 95 \% \mathrm{CI}, 0.06-0.61$ ), compound Kushen injection ( $\mathrm{OR}=0.21 ; 95 \% \mathrm{CI}, 0.07-0.63)$, Delisheng injection ( $\mathrm{OR}=0.18 ; 95 \% \mathrm{CI}, 0.05-0.66)$, Huachansu injection $(\mathrm{OR}=0.24 ; 95 \% \mathrm{CI}, 0.06-0.96)$, and Yadanziyouru injection $(\mathrm{OR}=0.21 ; 95 \% \mathrm{CI}, 0.05-0.83)$, and the differences between the groups were statistically significant. There were no significant differences in gastrointestinal reactions between the other CHIs (Table 3).

Based on the ranking results of reducing gastrointestinal reactions, the relative ranking of interventions was as follows: Shenqifuzheng injection $(95.63 \%)>$ Kangai injection $(71.31 \%)>$ Shenfu injection $(70.89 \%)>$ Kanglaite injection $(56.34 \%)>$ Xiaoaiping injection $(53.4 \%)>$ Huachansu injection $\quad(47.53 \%)>$ Shenmai injection $\quad(43.19 \%)$ $>$ Yadanziyouru injection $(41.5 \%)>$ Fufangkushen injection $(41.21 \%)>$ Aidi injection $(35.37 \%)>$ Delisheng injection $(32.92 \%)>$ TP only $(10.71 \%)$ (Figure 4$)$.

The SUCRA heat map of all the outcome indicators is shown in Figure 5. The warmer colors represent higher SUCRA values, which indicate that this intervention is more likely to be the best intervention.

3.7. Cluster Analysis. Cluster analysis was conducted on 11 interventions that reported both the clinical effective rate 


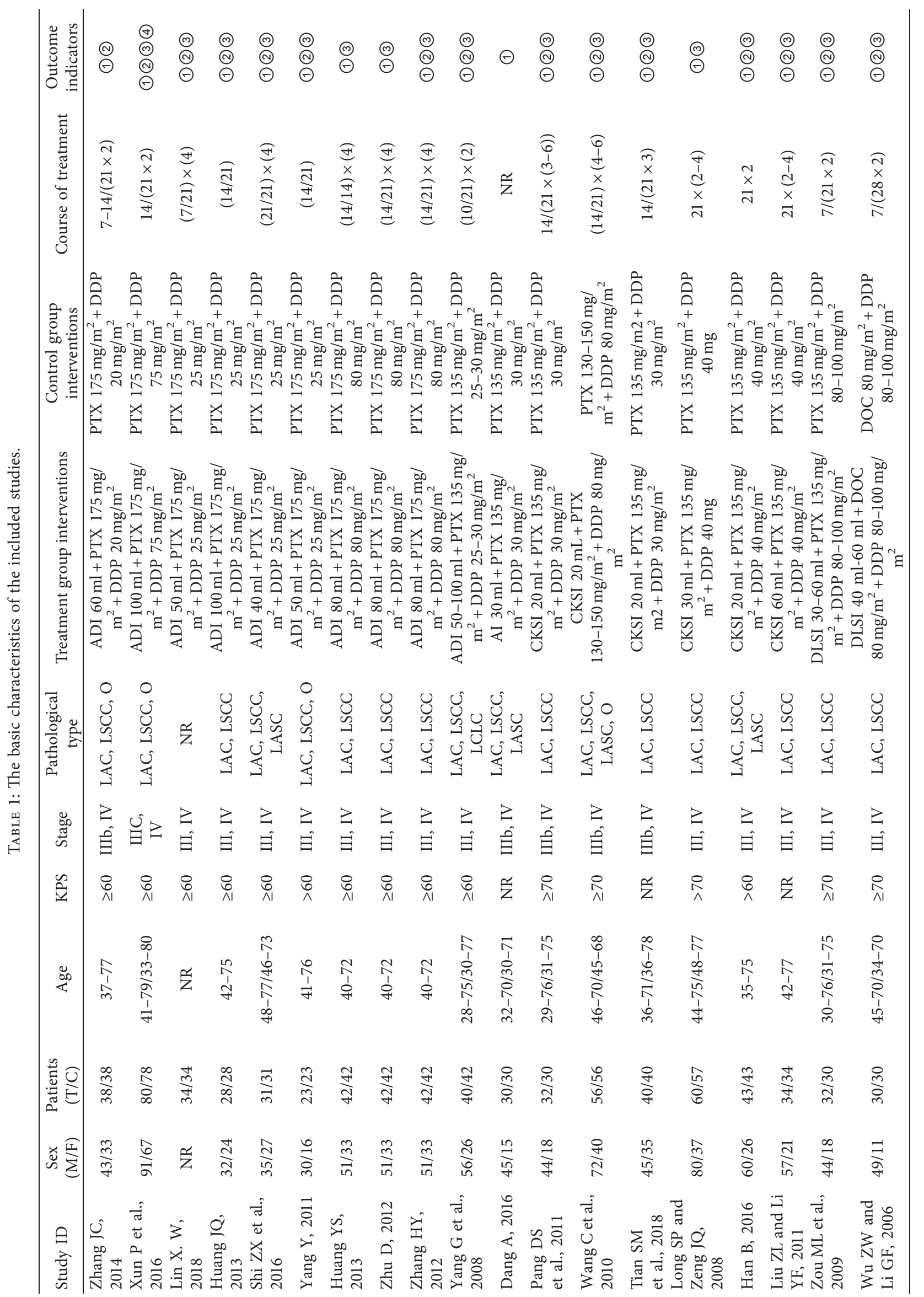




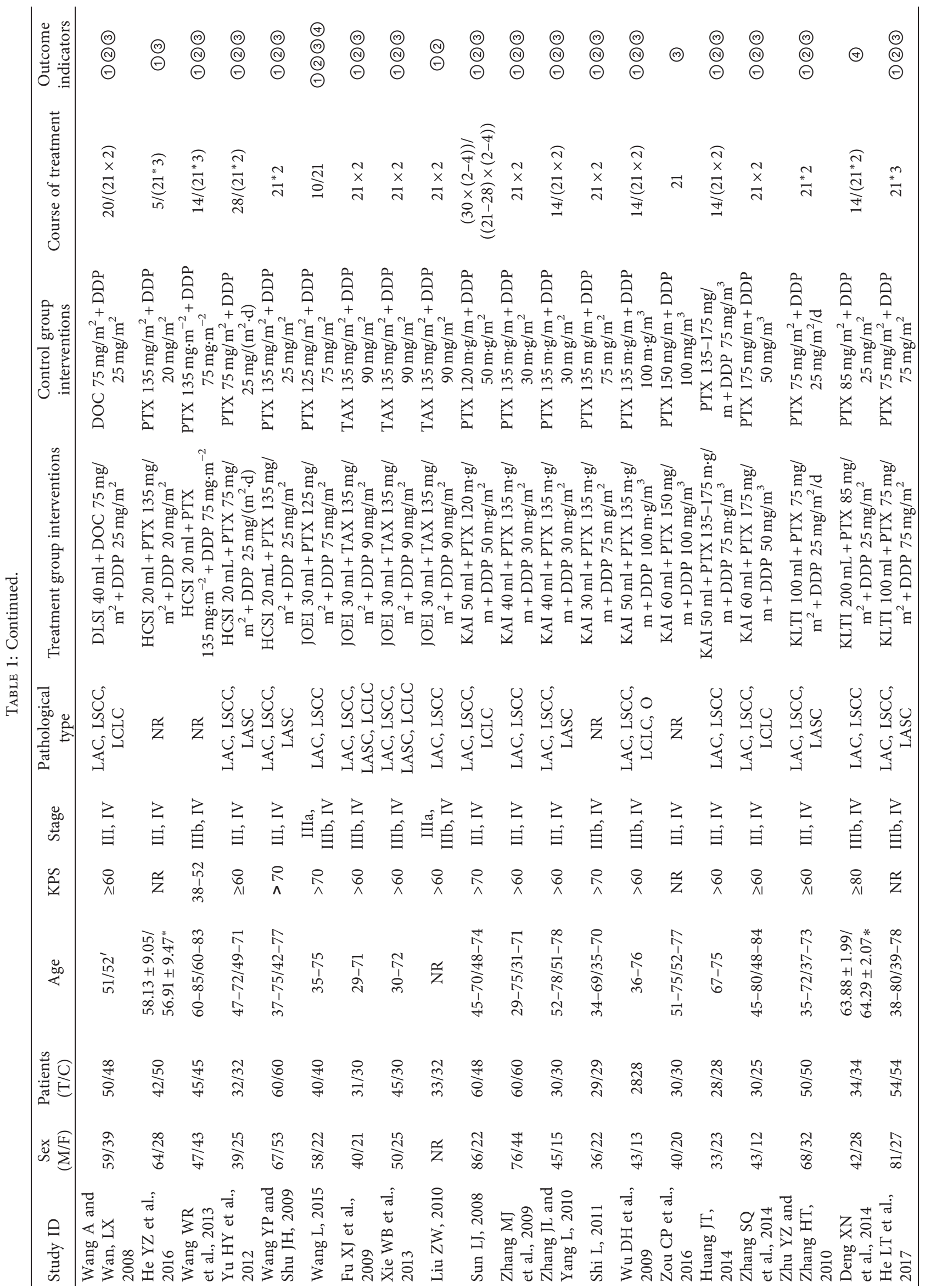




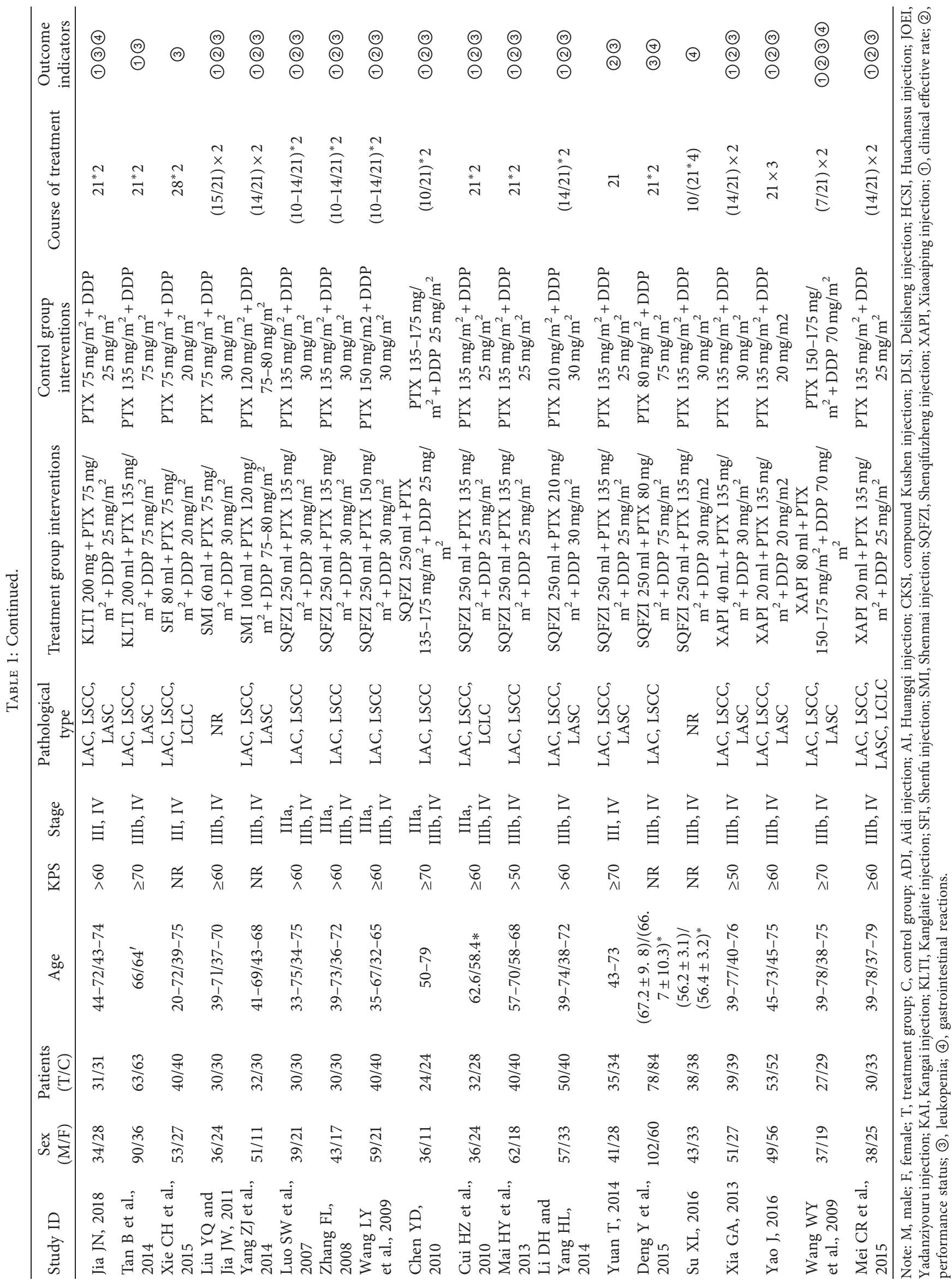




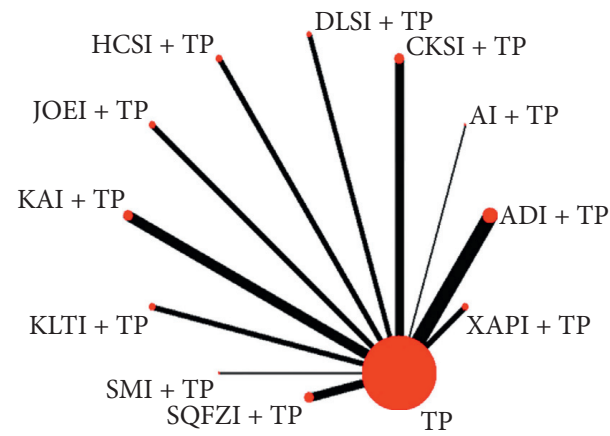

(a)

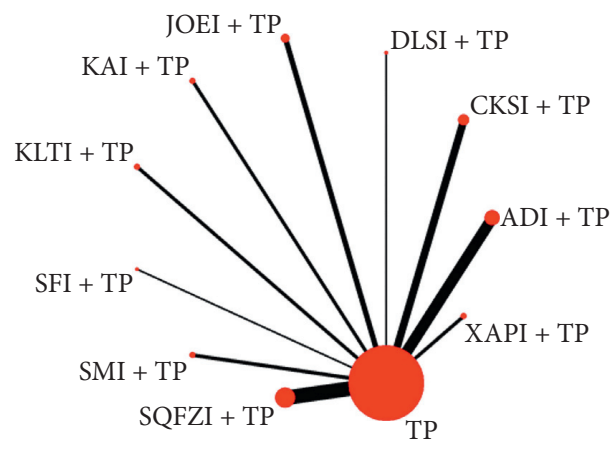

(c)

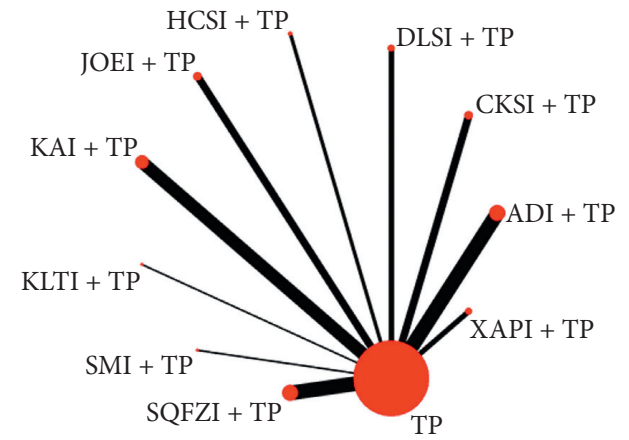

(b)

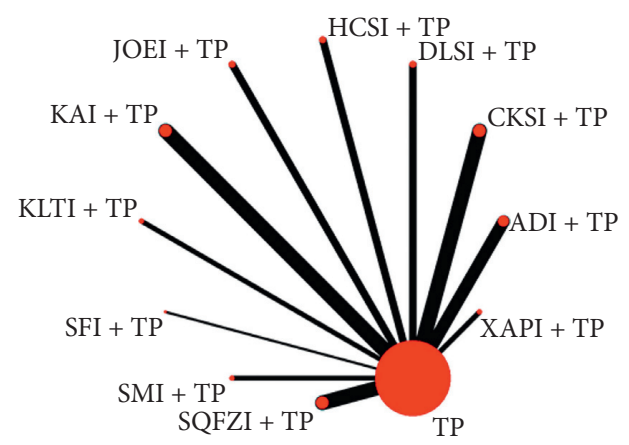

(d)

Figure 2: Network graph for different outcomes. (a) Clinical effective rate; (b) performance status; (c) leukopenia; and (d) gastrointestinal reactions. TP, paclitaxel plus cisplatin; XAPI, Xiaoaiping injection; ADI, Aidi injection; AI, Huangqi injection; CKSI, compound Kushen injection; DLSI, Delisheng injection; HCSI, Huachansu injection; JOEI, Yadanziyouru injection; KAI, Kangai injection; KLTI, Kanglaite injection; SMI, Shenmai injection; SQFZI, Shenqifuzheng injection; and SFI, Shenfu injection.

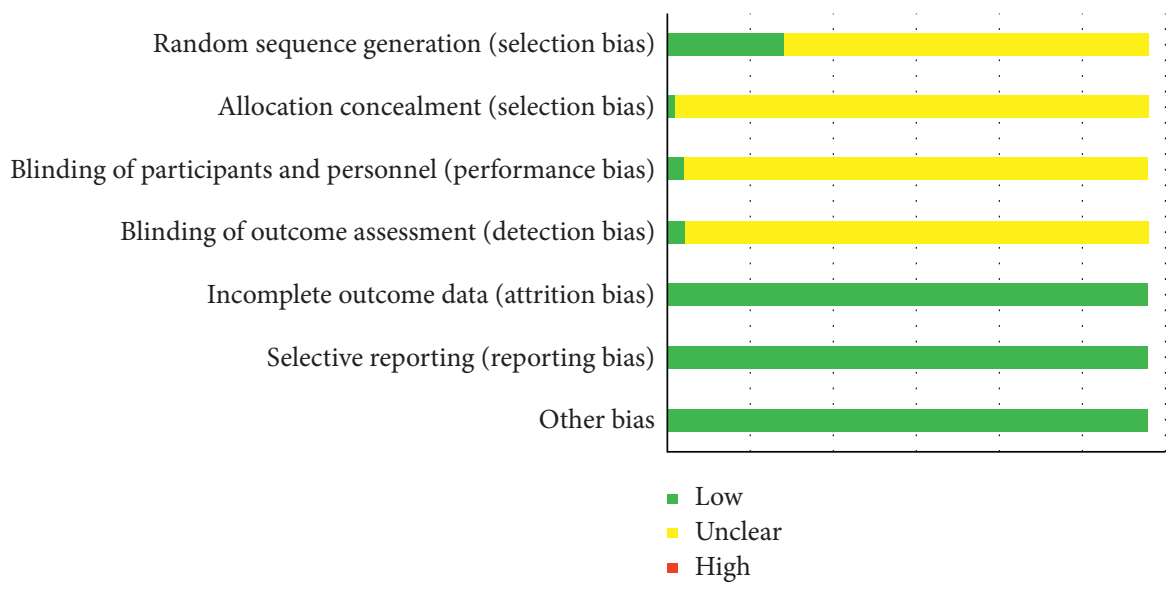

FIGURE 3: Assessment of the risk of bias.

and alleviation of leukopenia. Compared with other CHIs, Kangai injection combined with TP chemotherapy had the potential to be the best intervention according to the comprehensive ranking of the clinical effective rate and alleviation of leukopenia, while TP intervention only produced the worst result (Figure 6(a)). In regard to the cluster analysis of 10 interventions that reported both the improvement of performance status and alleviation of leukopenia, Kangai injection and Shenqifuzheng injection combined with TP chemotherapy had advantages in the comprehensive ranking, with TP only yielding the worst result (Figure 6(b)).

When cluster analysis was conducted with 10 interventions that reported the clinical effectiveness rate, improvement of performance status, and alleviation of leukopenia, Kangai injection combined with TP was dominant in the comprehensive ranking of all three outcome indicators, and TP only was evaluated as the 


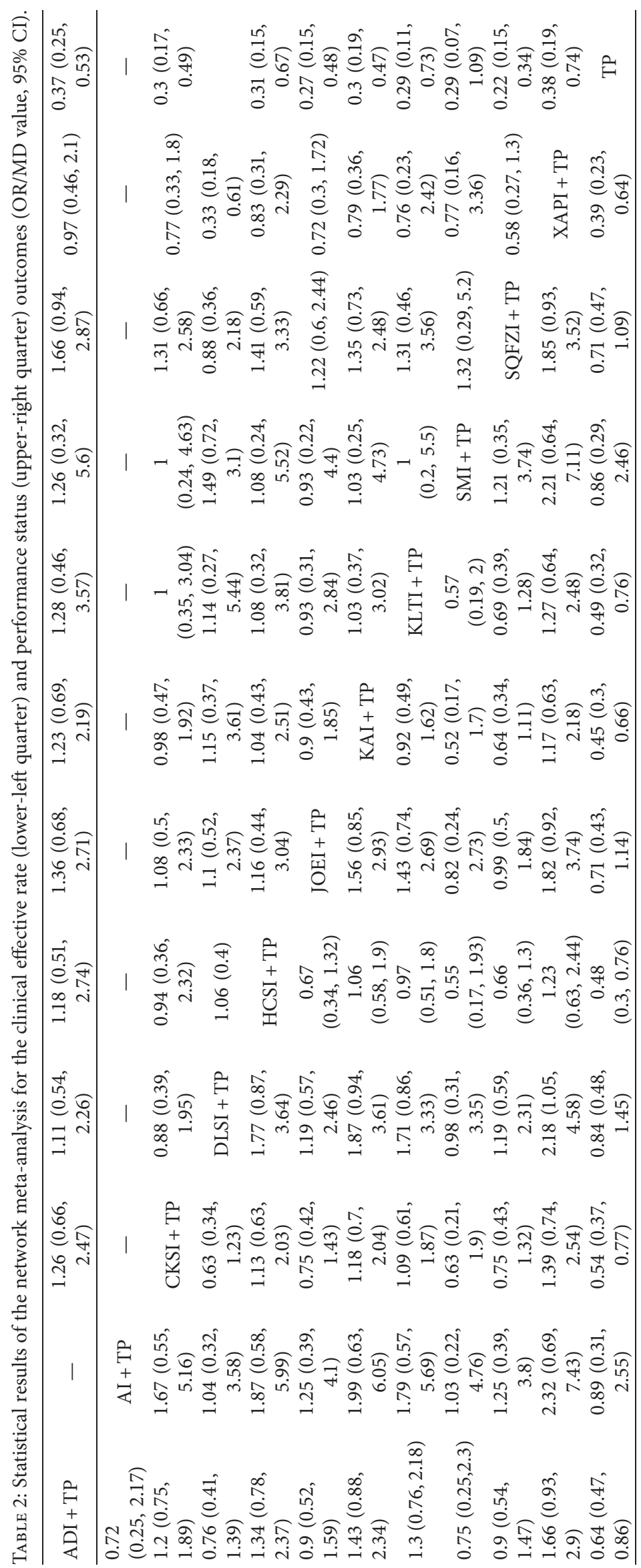



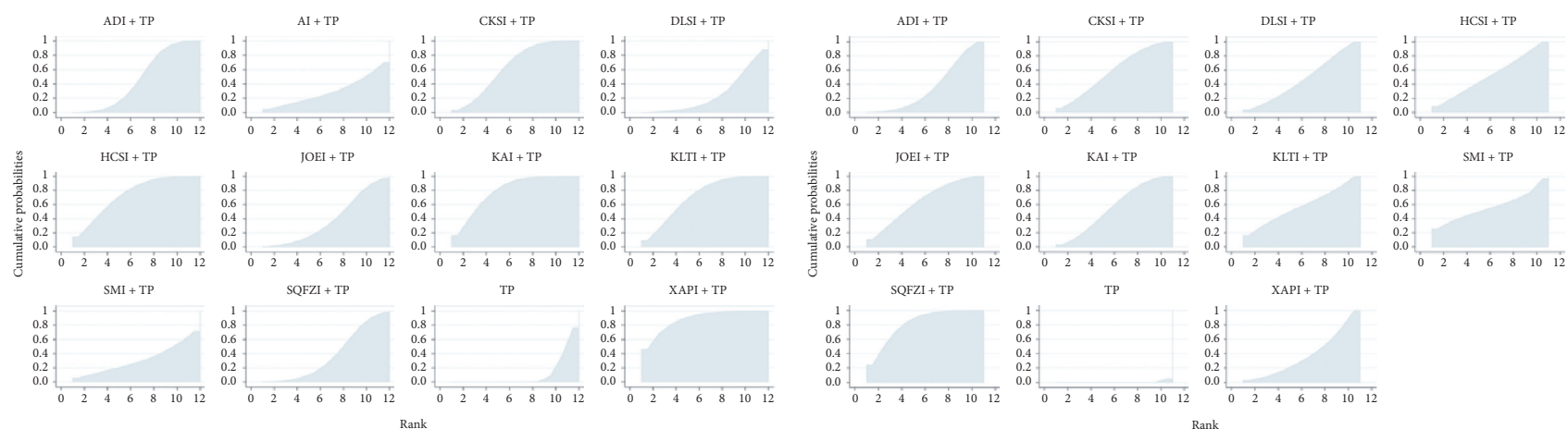

$\mathrm{SMI}+\mathrm{T}$
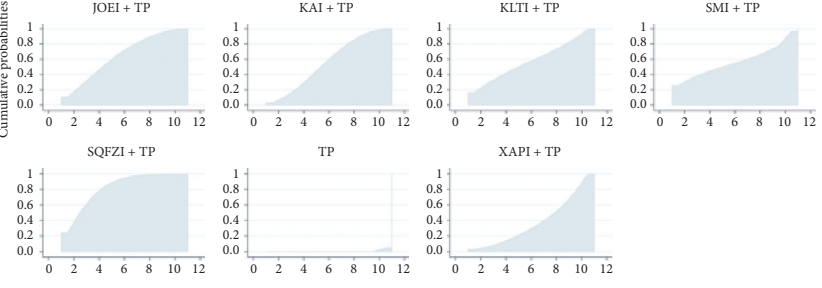

(a)
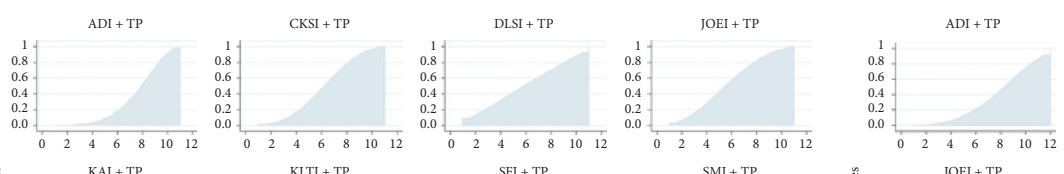

(b)
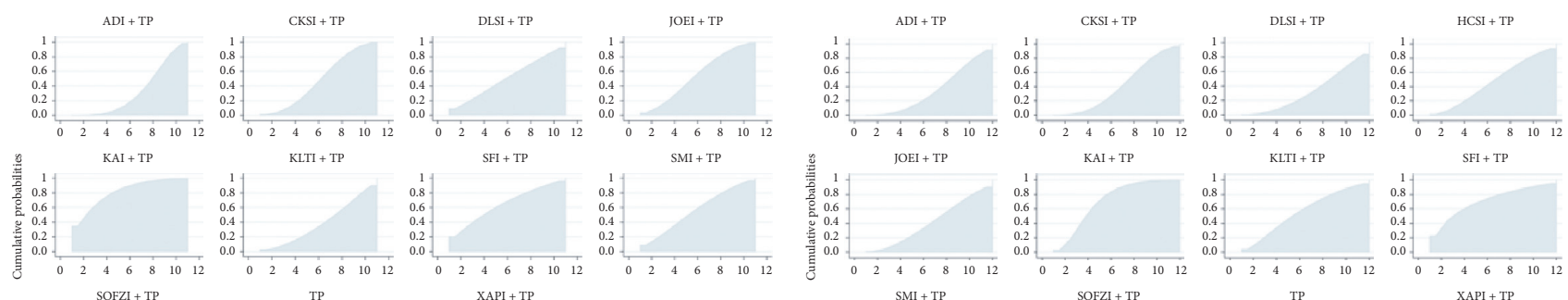

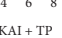
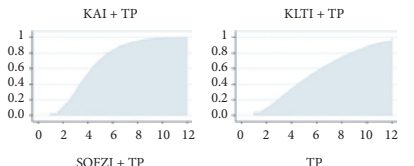

$\mathrm{SFI}+\mathrm{TP}$
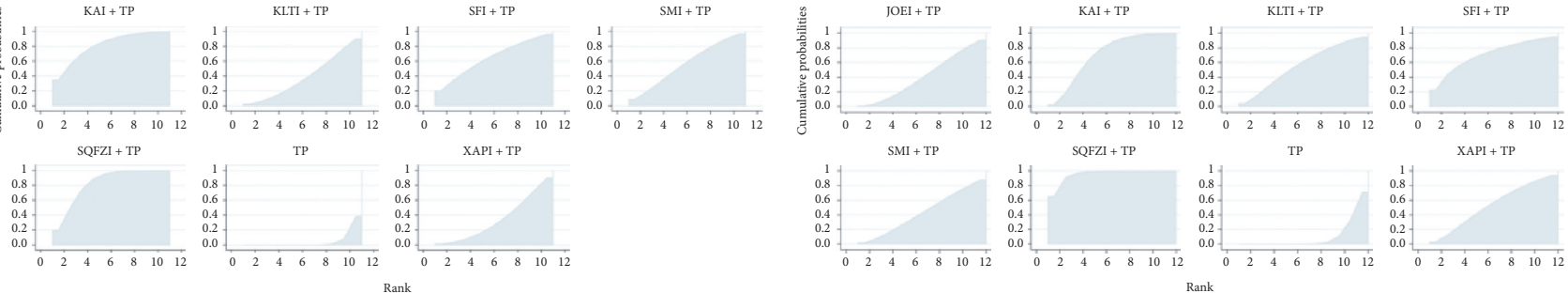

$\mathrm{XAPI}+\mathrm{TP}$

(c)

(d)

FIgURE 4: Surface under the cumulative ranking curves for all treatments. (a) Clinical effective rate; (b) performance status; (c) leukopenia; (d) gastrointestinal reactions. TP, paclitaxel plus cisplatin; XAPI, Xiaoaiping injection; ADI, Aidi injection; AI, Huangqi injection; CKSI, compound Kushen injection; DLSI, Delisheng injection; HCSI, Huachansu injection; JOEI, Yadanziyouru injection; KAI, Kangai injection; KLTI, Kanglaite injection; SMI, Shenmai injection; SQFZI, Shenqifuzheng injection; and SFI, Shenfu injection.

worst (Figure 6(c)). In regard to the cluster analysis of 11 interventions that reported the clinical effective rate, improvement of performance status, and alleviation of gastrointestinal reactions, Shenqifuzheng injection combined with TP chemotherapy had the potential to be the best intervention according to the comprehensive ranking, while TP only was considered the worst (Figure 6(d)). The cluster analysis conducted with 10 interventions that reported the clinical effectiveness rate, alleviation of leukopenia, and alleviation of gastrointestinal reactions indicated that Kangai injection and compound Kushen injection combined with TP chemotherapy had the potential to be the best intervention (Figure 6(e)). Kangai injection and compound Kushen injection combined with TP were dominant in the comprehensive ranking of the improvement of performance status, alleviation of leukopenia, and alleviation of gastrointestinal reactions (Figure 6(f)).

3.8. Publication Bias. Publication bias detection was performed using funnel plots for the clinical effective rate and performance status (Figure 7). There was incomplete symmetry in both funnel plots, and there were certain angles between the correction auxiliary line and the centerline, which indicated that this study has publication bias.

\section{Discussion}

This study compared the clinical efficacy and safety of 12 CHIs combined with TP in the treatment of NSCLC with the NMA method. The results are as follows. (1) In terms of the clinical effective rate, based on TP chemotherapy, Aidi injection, compound Kushen injection, Kangai injection, Kanglaite injection, Shenmai injection, and Yadanziyouru injection were significantly more effective than using TP chemotherapy alone. (2) In terms of performance status, based on TP chemotherapy, Aidi injection, compound Kushen injection, Huachansu injection, Yadanziyouru injection, Kangai injection, Kanglaite injection, Shenqifuzheng injection, and Xiaoaiping injection were significantly more effective than using TP chemotherapy alone. (3) In terms of ADRs/ADEs, based on TP chemotherapy, compound Kushen injection, Yadanziyouru injection, Kangai injection, and Shenqifuzheng injection were significantly more effective in reducing leukopenia than TP chemotherapy alone. Based on TP chemotherapy, Kangai injection and Shenqifuzheng injection were significantly more effective in reducing gastrointestinal reactions than TP chemotherapy alone. (4) In the cluster analysis, Kangai injection with TP chemotherapy was dominant in the comprehensive ranking of the clinical effective rate and alleviation of leukopenia. Based on TP chemotherapy, Kangai injection and Shenqifuzheng injection were dominant in the comprehensive 


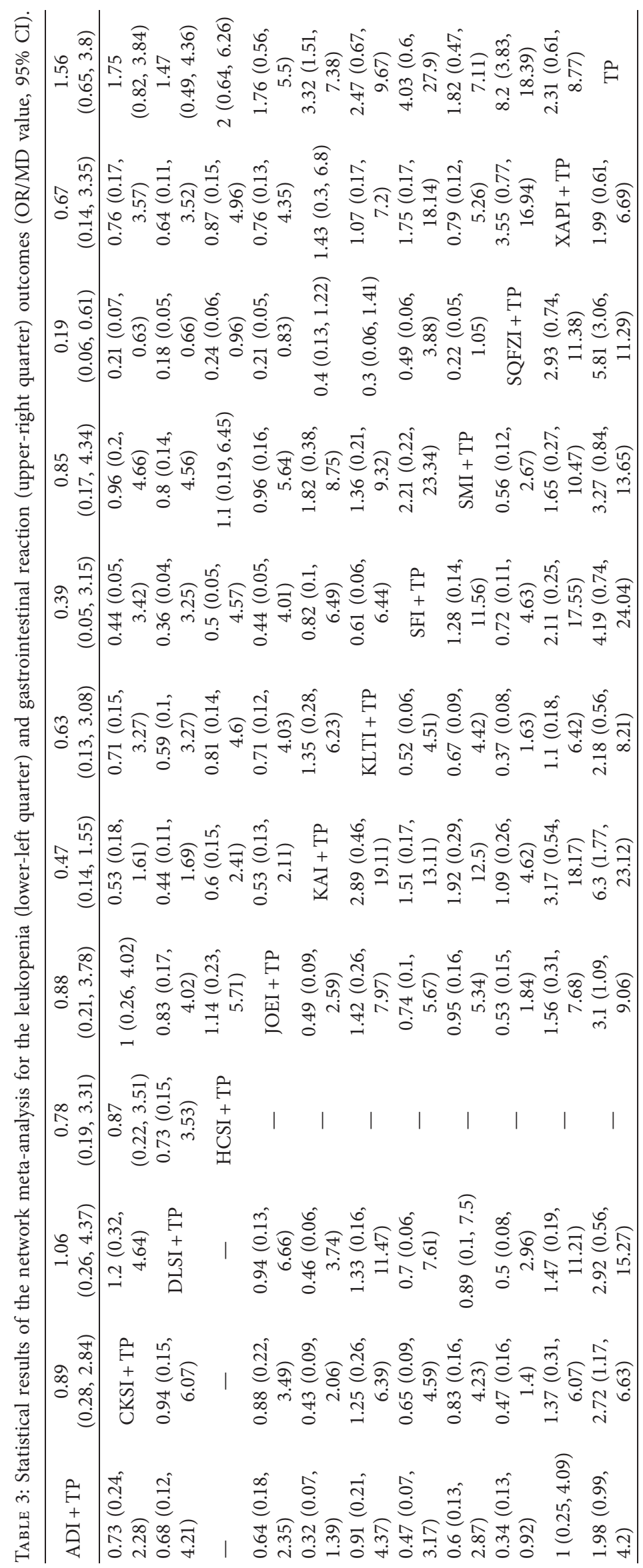




\begin{tabular}{|c|c|c|c|c|c|c|c|c|}
\hline Intervention & The clinical effective rate & Rank & Performance status & Rank & Leukopenia & Rank & Gastrointestinal & Rank \\
\hline$\overline{\mathrm{ADI}+\mathrm{TP}}$ & 47.07 & 6 & 36.61 & 10 & 33.14 & 10 & 35.37 & 10 \\
\hline $\mathrm{AI}+\mathrm{TP}$ & 19.72 & 10 & - & 0 & - & 0 & - & 0 \\
\hline $\mathrm{CKSI}+\mathrm{TP}$ & 64.13 & 5 & 58.32 & 3 & 48.32 & 7 & 41.21 & 9 \\
\hline $\mathrm{DLSI}+\mathrm{TP}$ & 25.98 & 11 & 47.58 & 8 & 51.07 & 6 & 32.92 & 11 \\
\hline $\mathrm{HCSI}+\mathrm{TP}$ & 73.95 & 3 & 25.59 & 7 & - & 0 & 47.53 & 6 \\
\hline $\mathrm{JOEI}+\mathrm{TP}$ & 39.35 & 7 & 64.72 & 2 & 54.25 & 5 & 41.5 & 8 \\
\hline $\mathrm{KAI}+\mathrm{TP}$ & 79.2 & , & 55.99 & 2 & 81.24 & 2 & 71.31 & 2 \\
\hline KLTI + TP & 71.62 & 2 & 57.79 & 6 & 39.05 & 8 & 56.34 & 4 \\
\hline $\mathrm{SMI}+\mathrm{TP}$ & 31.66 & 4 & 56.61 & 4 & 56.24 & 4 & 43.19 & 7 \\
\hline $\mathrm{SFI}+\mathrm{TP}$ & - & 9 & - & 5 & 64.46 & 3 & 70.89 & 3 \\
\hline SQFZI + TP & 38.13 & 8 & 81.82 & 1 & 82.63 & 1 & 95.63 & 1 \\
\hline $\mathrm{XAPI}+\mathrm{TP}$ & 88.2 & 1 & 37.54 & 9 & 34.74 & 9 & 53.4 & 5 \\
\hline $\mathrm{TP}$ & 10.99 & 12 & 0.4404 & 11 & 4.851 & 11 & 10.71 & 12 \\
\hline
\end{tabular}

FIGURE 5: Surface under the cumulative ranking curve (SUCRA) probability results. TP, paclitaxel plus cisplatin; XAPI, Xiaoaiping injection; ADI, Aidi injection; AI, Huangqi injection; CKSI, compound Kushen injection; DLSI, Delisheng injection; HCSI, Huachansu injection; JOEI, Yadanziyouru injection; KAI, Kangai injection; KLTI, Kanglaite injection; SMI, Shenmai injection; SQFZI, Shenqifuzheng injection; and SFI, Shenfu injection.

ranking of the improvement of performance status and alleviation of leukopenia.

NSCLC is a common malignant tumor that has the characteristics of high mortality and insignificant early symptoms. Due to the low rate of early diagnosis and the rapid development of the disease, most patients have already reached the advanced stage of local cancer or the tumor has already metastasized at the time of diagnosis. Conventional treatments for NSCLC include surgery, radiotherapy, and chemotherapy [8, 102-104]. However, while radiation or chemotherapy kills cancer cells, it can also cause serious toxic side effects, complications, and drug resistance. Therefore, many doctors and patients with NSCLC are actively exploring other complementary and alternative therapies for cancer treatment $[105,106]$. Traditional Chinese medicine has been used clinically for the treatment of cancer in China, Japan, and even other Asian countries for thousands of years. In recent years, Chinese medicine as a complementary and alternative medicine has been indicated to have unique advantages in improving the clinical symptoms of cancer patients, improving quality of life, enhancing body immunity, and extending survival. At the same time, the combined use of traditional Chinese medicine and conventional cancer treatment methods such as radiotherapy and chemotherapy also has a therapeutic effect of reducing toxicity and increasing efficacy [107]. According to relevant pharmacological studies of Yadanziyouru injection, which ranked dominantly in performance status, Brucea javanica, a plant in the Simaroubaceae family, has significant antimalarial and antitumor activities. In particular, fatty acid components, such as oleic acid, linoleic acid, palmitic acid, stearic acid, and arachidonic acid, have strong antitumor activity and have great killing effects on tumor cells [108-111]. The mechanism may be that they can selectively destroy the membranes and mitochondria of tumor cells, inhibit growth and DNA synthesis in tumor cells, block the cell cycle of cancer cells, improve immunity, and reverse the drug resistance to many drugs [112-115]. Shenqifuzheng injection consists of the traditional Chinese medicines Codonopsis and Astragalus. The two can be used together to promote blood circulation, eliminate blood stasis, and eliminate lumps. Modern pharmacological research shows that Codonopsis can enhance the body's immunity, increase the content of white blood cells, red blood cells, and hemoglobin, adjust gastrointestinal function, and resist fatigue. Astragalus enhances immune function and has a complete immune recovery effect on lymphocyte function in cancer patients [116-118].

At present, there are two related NMAs written in Chinese in the databases, both of which have systematically evaluated the clinical efficacy of CHIs combined with TP in the treatment of NSCLC. Compared with those two studies, the advantages of this study are reflected in the following aspects. (1) For the first time, this study used $R$ software to perform a multidimensional clustering analysis on the included outcome indicators, and the results were visualized through a three-dimensional stereogram. Different interventions were compared under multiple outcome indicators. (2) This study not only updated the search time and included the largest number of studies but also conducted a comprehensive search of $22 \mathrm{CHI}$ that have been used clinically for tumor treatment at this stage. The search terms used in this study were divided into three parts: NSCLC, CHIs, and RCTs. With a search strategy as the combination of mesh terms and free text, the literature retrieved for this study was comprehensive. (3) Only slight clinical heterogeneity was found among the included studies, as strict inclusion and exclusion criteria were established; the common intervention measure of the included RCTs was TP chemotherapy, and only the WHO evaluation criteria for solid tumor efficacy were adopted as the clinical efficacy evaluation criteria. (4) This study focused on not only important indicators to measure the therapeutic effect of cancer such as the clinical effective rate and improvement in performance status but also the incidence of ADRs/ADEs. Moreover, this study also 


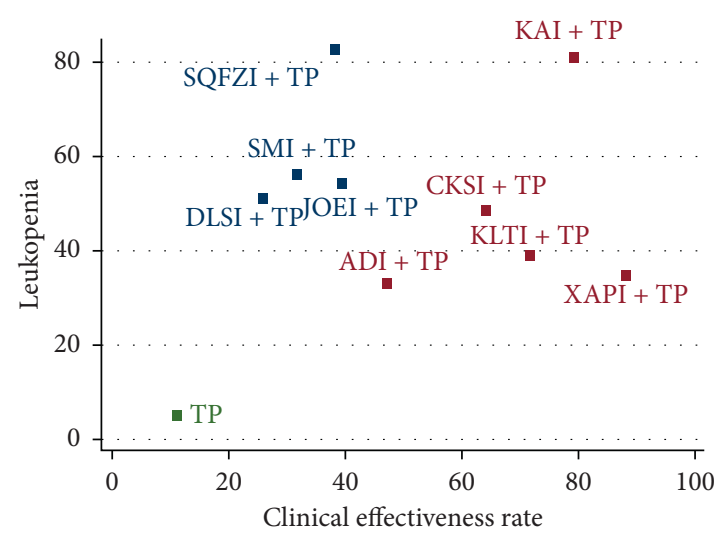

(a)

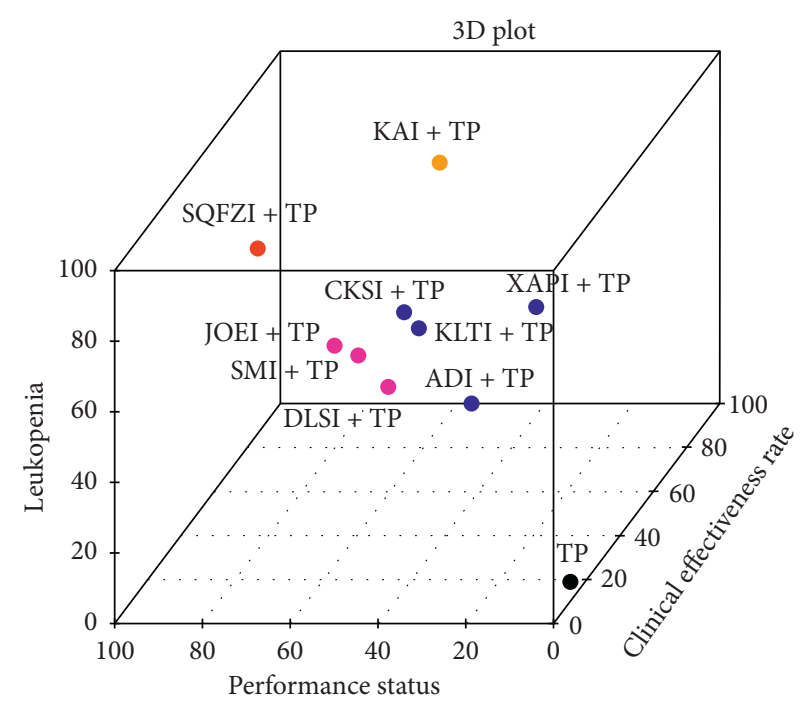

(c)

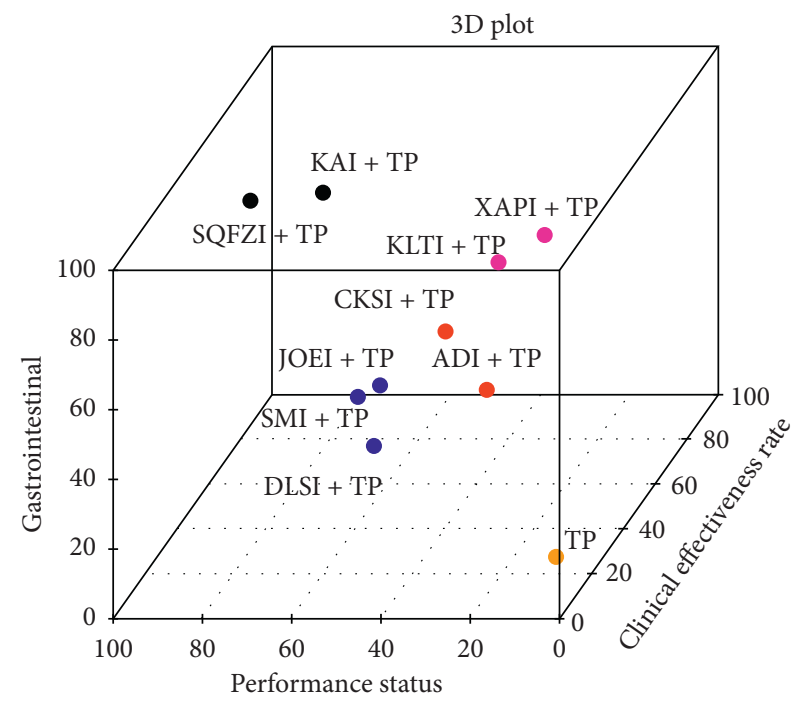

(e)

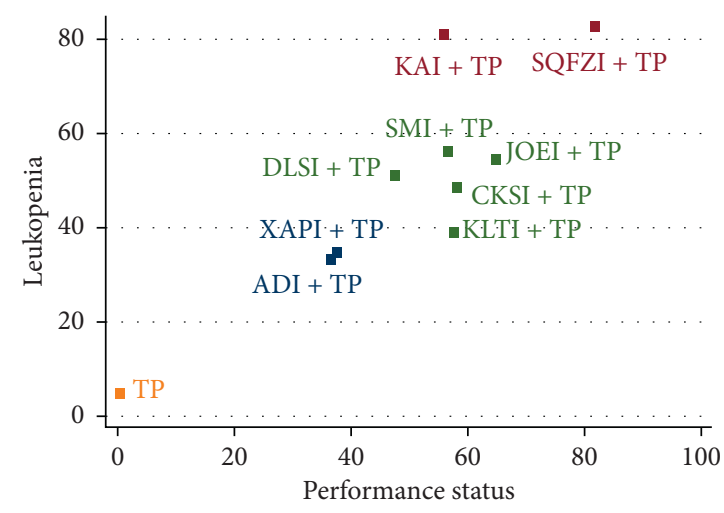

(b)

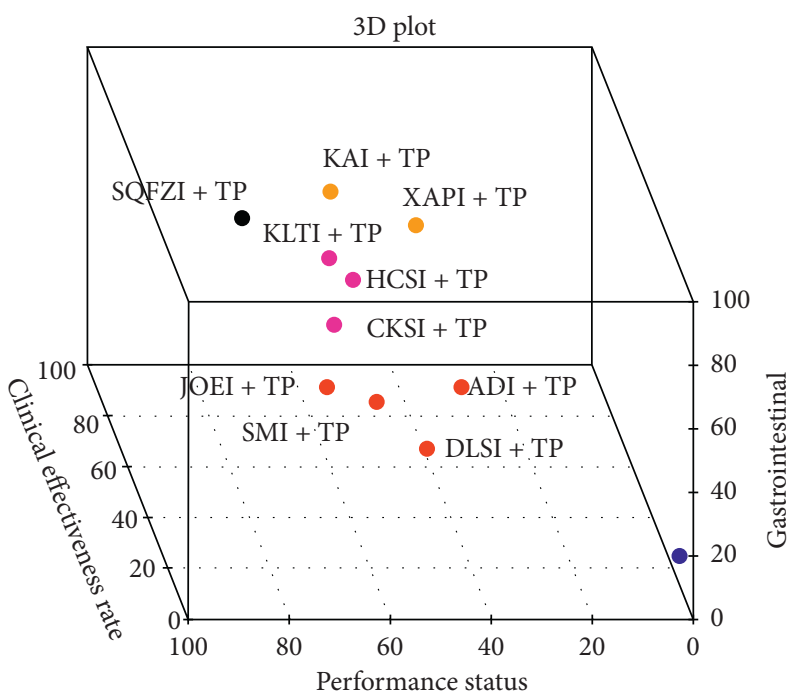

(d)

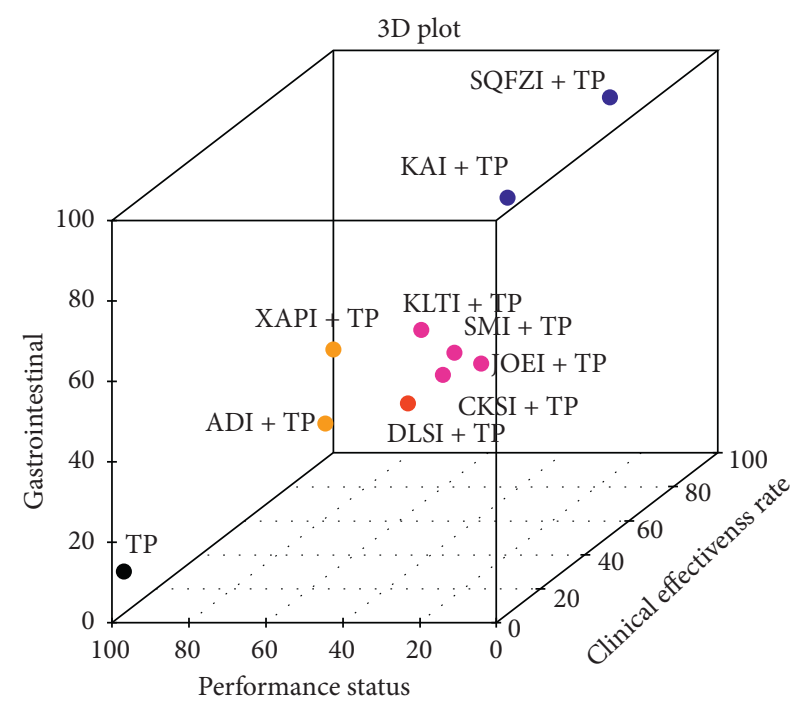

(f)

FIgURE 6: Cluster analysis plots. (a) Clinical effective rate and leukopenia; (b) performance status and leukopenia; (c) clinical effective rate, performance status, and leukopenia; (d) clinical effective rate, performance status, and gastrointestinal reactions; (e) clinical effective rate, leukopenia, and gastrointestinal reactions; and (f) performance status, leukopenia, and gastrointestinal reactions. TP, paclitaxel plus cisplatin; XAPI, Xiaoaiping injection; ADI, Aidi injection; AI, Huangqi injection; CKSI, compound Kushen injection; DLSI, Delisheng injection; HCSI, Huachansu injection; JOEI, Yadanziyouru injection; KAI, Kangai injection; KLTI, Kanglaite injection; SMI, Shenmai injection; SQFZI, Shenqifuzheng injection; and SFI, Shenfu injection. 


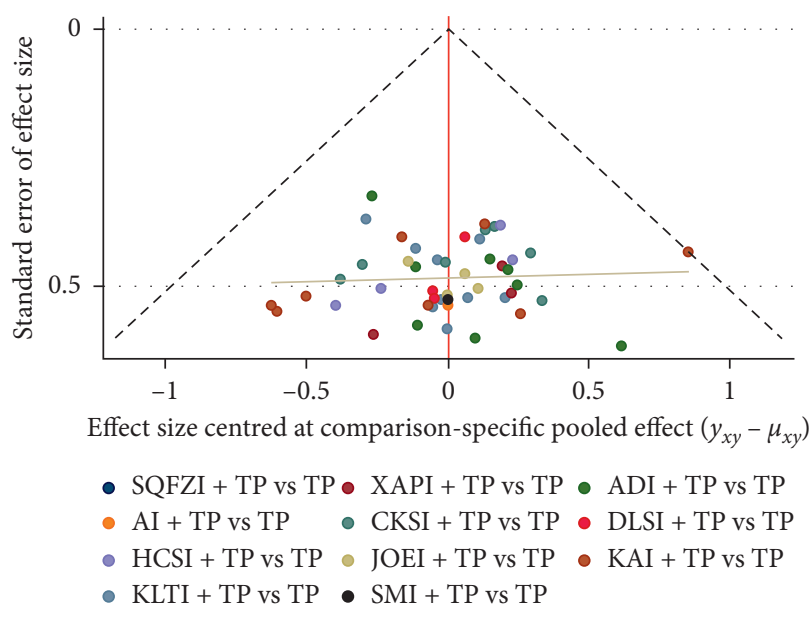

(a)

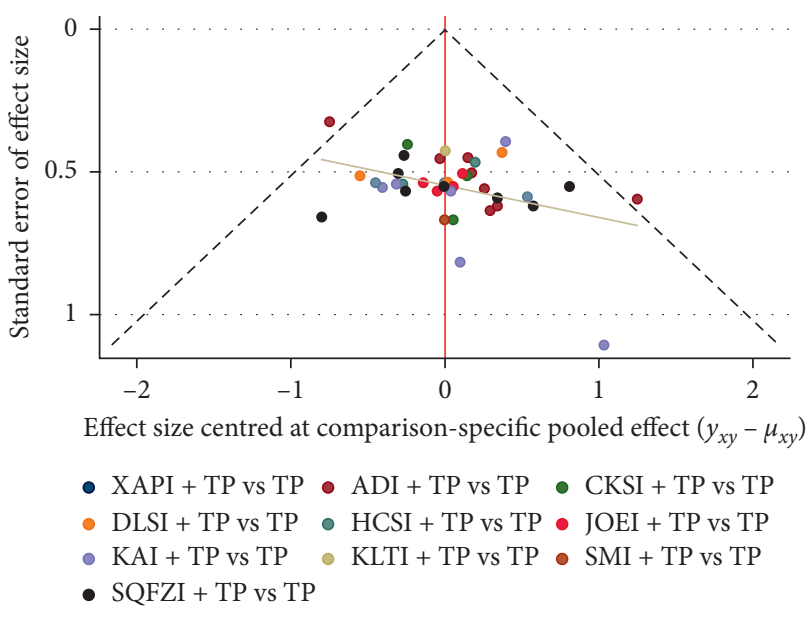

(b)

Figure 7: Funnel plots of publication bias. (a) Clinical effective rate; (b) performance status. TP, paclitaxel plus cisplatin; XAPI, Xiaoaiping injection; ADI, Aidi injection; AI, Huangqi injection; CKSI, compound Kushen injection; DLSI, Delisheng injection; HCSI, Huachansu injection; JOEI, Yadanziyouru injection; KAI, Kangai injection; KLTI, Kanglaite injection; SMI, Shenmai injection; and SQFZI, Shenqifuzheng injection.

performed cluster analysis and comprehensive ranking for the above outcome indicators.

\section{Limitations}

Nevertheless, there were still limitations in this study. First, the survival rate is critical in determining and judging the effectiveness of treatment for cancer patients; however, with insufficient information to perform NMA, this study did not evaluate such long-term endpoint outcome indicators. Next, the included studies did not conduct traditional Chinese medicine dialectical analysis. The systematic review should classify the data into different syndromes to provide clearer guidance for clinical practice. In addition, most of the RCTs in this systematic review had low methodological quality, small sample sizes, and incomplete descriptions of randomization and allocation. These RCTs also lack emphasis on the implementation of blinding methods and the approval of medical ethics, which may affect the reliability of the overall research. Therefore, we recommend that RCTs be registered in advance to ensure the transparency of the trial process and improve methodological quality. In addition, RCTs should be implemented in accordance with the latest clinical diagnostic and treatment guidelines. In view of the abovementioned limitations, the results of this study need to be verified by more well-designed, large-sample, multicenter RCTs.

\section{Conclusions}

In conclusion, the use of CHIs was associated with improved treatment performance and could be beneficial for patients with NSCLC compared to using TP alone. According to multidimensional cluster analysis, Kangai injection showed the best efficacy with respect to simultaneously increasing clinical efficacy and reducing ADRs/ADEs, followed by Shenqifuzheng injection and compound Kushen injection.
However, more direct comparison studies of two or more $\mathrm{CHI}$ are needed to further confirm the results.

\author{
Abbreviations \\ 95\% CI: $\quad 95 \%$ confidence interval \\ ADRs/ADEs: Adverse drug reactions/adverse drug events \\ CHIs: Chinese herbal injections \\ CR: $\quad$ Complete response \\ KPS: $\quad$ Karnofsky performance status \\ NMA: Network meta-analysis \\ NSCLC: Non-small-cell lung cancer \\ OR: Odds ratio \\ PD: $\quad$ Progressive disease \\ PR: $\quad$ Partial response \\ RCTs: $\quad$ Randomized controlled trials \\ SD: $\quad$ Stable disease \\ SUCRA: Surface under the cumulative ranking curve \\ TP: $\quad$ Paclitaxel plus cisplatin.
}

\section{Data Availability}

The datasets we generated during the analysis are not publicly available because the analysis process is the core of the results and cannot be made public. Requests to access the datasets of included studies can be met by checking out them in databases.

\section{Ethical Approval}

Ethical approval was not necessary in the current study because our study gathered data only from Embase, PubMed, the Cochrane Library, the China National Knowledge Infrastructure Database (CNKI), the Wan-fang Database, the CQVIP Database (VIP), and the China Biology Medicine disc (SinoMed) database, and this procedure 
did not address any patient's personal data or harm any patient.

\section{Conflicts of Interest}

The authors Bin Sun and Qianqian Zhang were employed by the company Jiangsu Jiuxu Pharmaceutical Co., Ltd. The remaining authors declare that the research was conducted in the absence of any commercial or financial relationships that could be construed as potential conflicts of interest.

\section{Authors' Contributions}

Mengwei Ni was involved in conceptualization, methodology, software, formal analysis, investigation, data curation, writing the original draft, visualization, and project administration. Haojia Wang was involved in methodology, formal analysis, investigation, writing the original draft, and visualization. Miaomiao Wang was involved in methodology and writing the review and editing. Wei Zhou was responsible for software and validation. Jiarui $\mathrm{Wu}$ was involved in conceptualization, resources, supervision, project administration, and funding acquisition. Bin Sun and Qianqian Zhang were responsible for validation and resources. Xiaotian Fan and Xinkui Liu were responsible for software. Dan Zhang and Xiaoguang Sheng were involved in writing the review and editing. Zhiwei Jing was responsible for resources. Jingyuan Zhang, Ziqi Meng, and Shuyu Liu conducted formal analysis. Siyu Guo and Shanshan Jia conducted investigation. Xiaomeng Zhang performed data Curation.

\section{Acknowledgments}

The authors acknowledge receipt of the following financial support for the research, authorship, and/or publication of this article: the National Natural Science Foundation of China (grant nos. 81473547 and 81673829) and Young Scientists Training Program of Beijing University of Chinese Medicine (grant no. BUCM-QNLJ 2019001).

\section{Supplementary Materials}

This file contains three parts, which includes items regarding the PRISMA checklist for network meta-analysis and corresponding pages of this study, the search strategy of traditional Chinese medicine injections in the PubMed database, and details about the product information of CHIs. (Supplementary Materials)

\section{References}

[1] F. Bray, J. Ferlay, I. Soerjomataram, R. L. Siegel, L. A. Torre, and A. Jemal, "Global cancer statistics 2018: GLOBOCAN estimates of incidence and mortality worldwide for 36 cancers in 185 countries," CA: A Cancer Journal for Clinicians, vol. 68, no. 6, pp. 394-424, 2018.

[2] J. Ferlay, I. Soerjomataram, R. Dikshit et al., "Cancer incidence and mortality worldwide: sources, methods and major patterns in GLOBOCAN 2012," International Journal of Cancer, vol. 136, no. 5, pp. E359-E386, 2015.
[3] R. L. Siegel, K. D. Miller, and A. Jemal, "Cancer statistics, 2017," CA: A Cancer Journal for Clinicians, vol. 67, no. 1, pp. 7-30, 2017.

[4] R. L. Siegel, K. D. Miller, and A. Jemal, "Cancer statistics, 2018," CA: A Cancer Journal for Clinicians, vol. 68, no. 1, pp. 7-30, 2018.

[5] V. Ernani, C. E. Steuer, and M. Jahanzeb, "The end of nihilism: systemic therapy of advanced non-small cell lung cancer," Annual Review of Medicine, vol. 68, no. 1, pp. 153-168, 2017.

[6] A. Pezzuto, M. Manicone, M. C. Scaini et al., "What information could the main actors of liquid biopsy provide? a representative case of non-small cell lung cancer (NSCLC)," Journal of Thoracic Disease, vol. 10, no. 7, pp. E570-E576, 2018.

[7] L. Crinò, W. Weder, J. Van Meerbeeck, E. Felip, and ESMO Guidelines Working Group, "Early stage and locally advanced (non-metastatic) non-small-cell lung cancer: ESMO clinical practice guidelines for diagnosis, treatment and follow-up," Annals of Oncology, vol. 21, no. Suppl5, pp. 103-115, 2010.

[8] S. G. Spiro and J. C. Porter, "Lung cancer-where are we today? current advances in staging and nonsurgical treatment," American Journal of Respiratory and Critical Care Medicine, vol. 166, no. 9, Article ID 11664, 2002.

[9] A. V. Nascimento, F. Gattacceca, A. Singh et al., "Biodistribution and pharmacokinetics ofMad2siRNA-loaded EGFR-targeted chitosan nanoparticles in cisplatin sensitive and resistant lung cancer models," Nanomedicine, vol. 11, no. 7, pp. 767-781, 2016.

[10] R. L. Siegel, K. D. Miller, and A. Jemal, "Cancer statistics," CA: A Cancer Journal for Clinicians, vol. 66, no. 1, pp. 10-29, 2016.

[11] Z. W. Chen and S. Lu, "Progress of the treatment of nonsmall cell lung cancer update in NCCN non-small cell lung cancer clinical practice guideline (version 2007)," Tumour Journal World, vol. 6, p. 223, 2007.

[12] Y. J. Zhang, F. Xu, J. C. Han, Y. J. Zhang, L. Li, and M. Zhao, "Clinical trial of pemetrexed combined with paclitaxel and cisplatin in the treatment of non small-cell lung cancer," Chinese Journal of Clinical Pharmacology (Chin.), vol. 32, no. 15, pp. 1396-1399, 2016.

[13] J. D. Hainsworth, A. A. Meluch, S. McClurkan et al., "Induction paclitaxel, carboplatin, and infusional 5-FU followed by concurrent radiation therapy and weekly paclitaxel/carboplatin in the treatment of locally advanced head and neck cancer: a phase II trial of the minnie pearl cancer research network," Cancer Journal, vol. 8, no. 4, pp. 311-321, 2016.

[14] Y. Chen, J. Li, S. Chen et al., "Nab-paclitaxel in combination with cisplatin versus docetaxel plus cisplatin as first-line therapy in non-small cell lung cancer," Scientific Reports, vol. 7, no. 1, pp. 10760-10767, 2017.

[15] W.-K. Yu, Z. Wang, C.-C. Fong et al., "Chemoresistant lung cancer stem cells display high DNA repair capability to remove cisplatin-induced DNA damage," British Journal of Pharmacology, vol. 174, no. 4, pp. 302-313, 2017.

[16] L. Amable, "Cisplatin resistance and opportunities for precision medicine," Pharmacological Research, vol. 106, pp. 27-36, 2016.

[17] D. Dumont, P. Dô, D. Lerouge et al., "Off-label use of crizotinib as a neoadjuvant treatment for a young patient when conventional chemotherapy gave No benefits in stage IIIA non-small cell lung cancer," American Journal of Case Reports, vol. 18, pp. 890-893, 2017. 
[18] T. Sugiyama, M. Mizuno, Y. Aoki et al., "A single-arm study evaluating bevacizumab, cisplatin, and paclitaxel followed by single-agent bevacizumab in Japanese patients with advanced cervical cancer," Japanese Journal of Clinical Oncology, vol. 47, no. 1, pp. 39-46, 2017.

[19] Y. Cheng and L. Zhang, "Research progress of antitumor mechanism of traditional Chinese medicine and drugs," China Pharmaceutical (Chin.), vol. 22, no. 17, pp. 103-104, 2013.

[20] Y. Yuan, "Anti-tumor effect of traditional Chinese medicine on immunologic intervention of $T$ lymphocytes," Chinese Journal of Cancer Biotherapy (Chin.), vol. 24, no. 2, pp. 189-193, 2017.

[21] H. B. Zhang, "Progress in traditional Chinese medicine treatment of non-small cell lung cancer," Hunan Journal of Traditional Chinese Medicine (Chin.), vol. 34, no. 2, pp. 155-156, 2018.

[22] T. T. Han, J. X. Zhou, M. Z. Ouyang, and D. H. Li, "Efficacy of TCM combined with chemotherapy in treatment of nonsmall cell lung cancer and its impact on survival time," Liaoning Journal of Traditional Chinese Medicine (Chin.), vol. 46, no. 4, pp. 729-732, 2019.

[23] F. Qi, A. Li, Y. Inagaki et al., "Chinese herbal medicines as adjuvant treatment during chemo-or radio-therapy for cancer," BioScience Trends (Chin.), vol. 6, no. 4, pp. 297-307, 2010.

[24] Q. M. Wang, L. M. Sun, and J. F. Xu, "Correlation between tongguanteng and its preparation Xiaoaiping against tumor," Clinical Misdiagnosis Misther (Chin.), vol. 30, no. 12, pp. 102-105, 2017.

[25] A. Zheng, T. Li, Y. Chen, J. Fang, Y. Zhang, and J. Feng, "Inhibitory effect of a Chinese medicine Xiaoaiping combined with cisplatin on the proliferation, invasion and apoptosis in ovarian cancer HO-8910 PM cells in vitro and in vivo," Zhonghua Zhong Liu Za Zhi (Chin.), vol. 38, no. 1, pp. 11-16, 2016.

[26] D. Li, C. Li, Y. Song et al., "Marsdenia tenacssima extract and its functional components inhibits proliferation and induces apoptosis of human burkitt leukemia/lymphoma cells in vitro and in vivo," Leukemia Lymphoma, vol. 18, pp. 1-10, 2015.

[27] E. Koumtebaye, N. Su, W.-F. Hu, S.-S. Lin, L. Sun, and S.-T. Yuan, "Antitumor activity of Xiaoaiping injection on human gastric cancer SGC-7901 cells," Chinese Journal of Natural Medicines, vol. 10, no. 5, pp. 339-346, 2012.

[28] S. Y. Han, W. X. Zheng, X. R. He et al., "Xiaoaiping injection combined with gefitinib inhibits resistant non-small cell lung cancer xenografts H460 and H1975," Chinese Journal of Pharmacology and Toxicology (Chin.), vol. 30, no. 1, pp. 44-52, 2016.

[29] Y.-J. Hu, X.-L. Shen, H.-L. Lu et al., "Tenacigenin B derivatives reverse $P$-glycoprotein-mediated multidrug resistance inHepG2/Dox cells," Journal of Natural Products, vol. 71, no. 6, pp. 1049-1051, 2008.

[30] Z. Huang, Y. Wang, J. Chen, R. Wang, and Q. Chen, "Effect of Xiaoaiping injection on advanced hepatocellular carcinoma in patients," Journal of Traditional Chinese Medicine, vol. 33, no. 1, pp. 34-38, 2013.

[31] G. Salanti, C. Del Giovane, A. Chaimani, D. M. Caldwell, and P. Higgins, "Evaluating the quality of evidence from a network meta-analysis," PLoS One, vol. 9, Article ID e99682, 2014.

[32] B. Hutton, G. Salanti, D. M. Caldwell et al., "The PRISMA extension statement for reporting of systematic reviews incorporating network meta-analyses of health care interventions: checklist and explanations," Annals of Internal Medicine, vol. 162, no. 11, pp. 777-784, 2015.

[33] R. Sylvester, WHO Handbook for Reporting Results of Cancer Treatment WHO offset Publication \#48 World Health Organization, World Health Organization, Geneva, Switzerland, 1979.

[34] People's Republic of China Department of Health Management, Guidelines for the Diagnosis and Treatment of Cancer, Pecking Union Medical College Press, Beijing, China, 1991.

[35] M. Tarsilla, "Cochrane handbook for systematic reviews of interventions," The Cochrane Collaboration, vol. 5, p. S38, 2011.

[36] S. Shim, B. H. Yoon, I. S. Shin, and J. M. Bae, "Network metaanalysis: application and practice using stata," Epidemiology and Health, vol. 39, Article ID e2017047, 2017.

[37] G. Rücker and G. Schwarzer, "Ranking treatments in frequentist network meta-analysis works without resampling methods," BMC Medical Research Methodology, vol. 15, pp. 1-9, 2015.

[38] L. Trinquart, N. Attiche, A. Bafeta, R. Porcher, and P. Ravaud, "Uncertainty in treatment rankings: reanalysis of network meta-analyses of randomized trials," Annals of Internal Medicine, vol. 164, no. 10, pp. 666-673, 2016.

[39] W. Cai, Y. Gu, H. Cui et al., "The efficacy and safety of mainstream medications for patients with cDMARD-naïve rheumatoid arthritis: a network meta-analysis," Frontiers in Pharmacology, vol. 9, pp. 1-9, 2018.

[40] L. Chang and R. Guo, "Comparison of the efficacy among multiple chemotherapeutic interventions combined with radiation therapy for patients with cervix cancer after surgery: a network meta-analysis," Oncotarget, vol. 8, no. 30, pp. 49515-49533, 2017.

[41] A. A. Veroniki, C. Soobiah, A. C. Tricco, M. J. Elliott, and S. E. Straus, "Methods and characteristics of published network meta-analyses using individual patient data: protocol for a scoping review," BMJ Open, vol. 5, Article ID e007103, 2015.

[42] L. Trinquart, G. Chatellier, and P. Ravaud, “Adjustment for reporting bias in network meta-analysis of antidepressant trials," BMC Medical Research Methodology, vol. 12, pp. 1-11, 2012.

[43] U. Krahn, H. Binder, and J. König, "Visualizing inconsistency in network meta-analysis by independent path decomposition," BMC Medical Research Methodology, vol. 14, pp. 1-12, 2014.

[44] J. C. Zhang, "Clinical observation of aidi combined with chemotherapy for advanced non-small cell lung cancer," China Health and Nutrition (Chin.), vol. 24, no. 2, pp. 1036-1037, 2014.

[45] P. Xun, L. Han, L. B. Zhao et al., "Observation on efficacy of aidi injections in adjuvant treatment of advanced non-small cell lung cancer," Evaluation and Analysis of Drug Use Hospitals China (Chin.), vol. 16, no. 9, pp. 1164-1166, 2016.

[46] X. W. Lin, "Clinical observation of aidi injection combined with paclitaxel and cisplatin regimen in the treatment of advanced non-small cell lung cancer," Medical Innovation of China (Chin.), vol. 15, no. 3, pp. 62-65, 2018.

[47] J. Q. Huang, "The study of aidi injection combined with PP chemotherapy in treating advanced non-small cell lung cancer," Jiankang Bidu (Chin.), no. 1, pp. 3-14, 2013.

[48] Z. X. Shi, J. Zhang, B. B. Zhang, and Z. Ma, "Clinical observation of aidi injection combined with TP regimen in the 
treatment of non-small cell lung cancer," China Pharmacy (Chin.), vol. 27, no. 23, pp. 3234-3237, 2016.

[49] Y. Yang, "Clinical observation of aidi injection combined with TP chemotherapy in the treatment of 46 cases of nonsmall cell lung cancer," Guide China Medicine (Chin.), vol. 9, no. 24, p. 105, 2011.

[50] Y.S. Huang, "Clinical observation of aidi injection combined with chemotherapy for non-small cell lung cancer," Medical and Aesthetic Cosmetology (Chin.), no. 3, pp. 168-193, 2013.

[51] D. Zhu, "Aidi injection combined with paclitaxel plus cisplatin chemotherapy in the treatment of advanced non-small cell lung cancer," Medical and Aesthetic Cosmetology (Chin.), vol. 20, no. 9, p. 191, 2012.

[52] H. Y. Zhang, "Clinical observation of aidi injection combined with chemotherapy for non-small cell lung cancer," Medical and Aesthetic Cosmetology (Chin.), vol. 21, no. 8, pp. 231-232, 2012.

[53] G. Yang, H. X. Jiang, and B. Wang, "Therapeutic effect of aidi injection combined with chemotherapy on 40 cases of advanced non-small cell lung cancer," Clinical Focus (Chin.), vol. 23, no. 22, pp. 1656-1657, 2008.

[54] A. Dang, "Effect of huangqi injection combined with chemotherapy on quality of life in patients with advanced nonsmall cell lung cancer," Journal Today Health (Chin.), vol. 15, no. 1, p. 15, 2016.

[55] D. S. Pang, Y. Xu, X. Y. Wang, and Z. G. Song, "Clinical observation of advanced non-small-cell lung cancer treated with TP regimen combined with fuFang kushen injection," Chinese Journal of Clinical Oncology and Rehabilitation (Chin.), vol. 18, no. 1, pp. 46-48, 2011.

[56] C. Wang, L. Wang, and Q. Shu, "Clinical observation of matrine injection combined with TP project in treatment of non small cell lung cancer," Clinics in Laboratory Medicine (Chin.), vol. 7, no. 16, pp. 1681-1682, 2010.

[57] S. M. Tian, J. J. Wu, and Y. P. Wu, "Effect of compound kushen injection combined with TP scheme on clinical symptoms, toxic and side effect and quality of life for patients with advanced lung cancer," Journal of Sichuan Traditional Chinese Medicine (Chin.), vol. 36, no. 2, pp. 213-215, 2018.

[58] S. P. Long and J. Q. Zeng, "Efficacy of fufang kushen injection combined with TP in the treatment of non-small cell lung cancer," Jiangxi Medical Journal (Chin.), vol. 43, no. 9, pp. 919-920, 2008.

[59] B. Han, "Clinical study of compound matrine in chemotherapy of advanced non small cell lung cancer," Medical Informatics (Chin.), vol. 29, no. 30, pp. 97-98, 2016.

[60] Z. L. Liu and Y. F. Li, "Observation on the curative effect of high-dose compound kushen injection on 34 cases of nonsmall cell lung cancer," Shandong Medical Journal (Chin.), vol. 51, no. 5, p. 78, 2011.

[61] M. L. Zou, Q. Y. Li, and F. X. Zuo, "The efficacy of delisheng combined with TP chemotherapy in treatment of advanced non-small cell lung cancer," Journal of Modern Oncology (Chin.), vol. 17, no. 2, pp. 274-275, 2009.

[62] Z. W. Wu and G. F. Li, "The efficacy of delisheng combined with TP chemotherapy in treatment of advanced non-small cell lang cancer," Journal of Modern Oncology (Chin.), vol. 14, no. 11, pp. 1444-1445, 2006.

[63] A. Wang and L. X. Wan, "The clinical efficacy of DeLiSheng injection combined with TIP regimen for advanced nonsmall cell lung cancer," China Practice Medicine (Chin.), vol. 3, no. 21, pp. 63-64, 2008.
[64] Y. Z. He, Y. H. Liu, and Z. J. Dai, "Efficacy evaluation of huachansu injection in advanced NSCLC," Inner Mongolia Medical Journal, vol. 48, no. 2, pp. 233-235, 2016.

[65] W. R. Wang, B. Hong, and K. Li, "Evaluation of cinobutacini injection in the adjuvant treatment of patients with advanced no-small-cell lung cancer," Journal of Clinical Pulmonary Medicine (Chin.), vol. 18, no. 2, pp. 203-204, 2013.

[66] H. Y. Yu, S. Y. Gao, and Y. X. Hao, "Study of huanchansu injection combined with TP regimen in the treatment of patients with advanced non-small cell lung cancer," Practice Journal of Cancer (Chin.), vol. 27, no. 1, pp. 55-57, 2012.

[67] Y. P. Wang and J. H. Shu, "Therapeutics effect observation of bufonin injection combined with chemotherapeutics on the primary NSCLC," World Journal of Tumor (Chin.), vol. 8, no. 3, pp. 183-184, 2009.

[68] L. Wang, "Observation of short-term efficacy of brucea javanica oil emulsion injection combined with chemotherapy in the treatment of advanced non-small cell lung cancer," Asia-Pacific Traditional Medicine (Chin.), vol. 11, no. 15, pp. 121-122, 2015.

[69] X. J. Fu, S. Z. Fu, and G. H. Yang, "Clinical obseration of brucea javanica oil emulsion combined chemotherapy on treating non-small cell lung cancer," Clinical Journal of Medical Officer (Chin.), vol. 37, no. 4, pp. 635-637, 2009.

[70] W. B. Xie, L. H. Luo, C. Hou, F. L. Song, and R. Y. Xie, "Clinical observation of brucea javanica oil emulsion injection combined with TP chemotherapy regimen in the treatment of non-small cell lung cancer," Chinese Journal of Clinical Rational Drug Use (Chin.), vol. 6, no. 16, pp. 72-73, 2013.

[71] Z. W. Liu, "Obseration of brucea javanica oil emulsion combined chemotherapy on treating non-mall cell lung cancer," Chinese Manipulation \& Rehabilitation Medicine (Chin.), vol. 1, no. 4, p. 41, 2010.

[72] L. J. Sun, "Clinical observation on treating non small cell lung cancer with kang'ai injection and chemotherapy," Chinese Health Care (Chin.), vol. 16, no. 3, pp. 1-2, 2008.

[73] M. J. Zhang, C. F. Zuo, and L. Q. Chai, "Clinical observation of kang'ai injection combined with chemotherapy in the treatment of advanced and advanced non-small cell lung cancer," Shanghai Medical and Pharmaceutical Journal (Chin.), vol. 30, no. 5, pp. 213-215, 2009.

[74] J. L. Zhang and L. Yang, "Clinical observation of combined kangai injection with TP in the treatment of advanced nonsmall cell lung cancer," Journal of Modern Oncology (Chin.), vol. 18, no. 6, pp. 1132-1134, 2010.

[75] L. Shi, "Observation of clinical effects of Kang'ai injection in treatment of advanced non-small cell lung cancer with TP chemotherapy regimen," China Practical Medical (Chin.), vol. 6, no. 29, pp. 151-152, 2011.

[76] D. H. Wu, N. J. Chen, Y. Y. Chen, and Y. Q. Lai, "Kang'ai injection combined with paclitaxel and DDP chemotherapy for 28 patients with advanced non-small cell lung cancer," Fujian Journal of Traditional Chinese Medicine (Chin.), vol. 40, no. 5, pp. 20-21, 2009.

[77] C. P. Zou, J. H. Yan, Y. Guo, Y. Q. Zhang, Y. C. Zhao, and Y. H. An, "Observing on the clinical effect of Kang'ai injection on patients with lung cancer," Pharmacological and Clinical Chinese Materia Medica (Chin.), vol. 32, no. 2, pp. 204-206, 2016.

[78] J. T. Huang, "Observation on the efficacy of Kang'ai injection combined with chemotherapy in the treatment of elderly patients with advanced non-small cell lung cancer," Contemporary Medicine (Chin), vol. 20, no. 6, pp. 145-146, 2014. 
[79] S. Q. Zhang, L. F. Lin, H. B. Zheng, X. H. Qiu, X. W. Zhuang, and Z. M. Chen, "Clinical efficacy of Kang'ai injection combined with chemotherapy in treatment of advanced lung cancer: an observation of 55 cases," China Medicine and Pharmacy (Chin.), vol. 4, no. 12, pp. 62-64, 2014.

[80] Y. Z. Zhu and H. T. Zhang, "Therapeutic effect of kanglaite injection combined with TP regimen on 100 cases of advanced non-small cell lung cancer," Journal of Modern Oncology (Chin.), vol. 18, no. 8, pp. 1569-1571, 2010.

[81] X. N. Deng, S. F. Zhao, H. J. Zhao, Y. L. Yan, L. Y. He, and H. Z. Zhang, "Regulatory effect of kanglaite injection on immune function in patients with non small cell lung cancer treated with chemotherapy," Modern Journal of Integrated Traditional Chinese and Western Medicine (Chin.), vol. 34, pp. 3767-3773, 2014.

[82] L. T. He, X. Y. Wu, X. Z. Yu, and H. C. Chen, "Analysis of kanglaite injection for improving clinical effect and quality of life in patients with advanced lung cancer," Modern Practice of Medicine (Chin.), vol. 29, no. 3, pp. 329-330, 2017.

[83] J. N. Jia, "Effect of kanglaite injection on immune function and adverse reactions in advanced NSCLC patients with chemotherapy," Journal of Community Medicine (Chin.), vol. 16, no. 5, pp. 11-13, 2018.

[84] B. Tan, F. R. Wu, Y. Bai, M. Chen, and Y. Wan, "Effect of coix seed oil injection in the treatment for advanced non-small cell lung cancer," Journal of Chinese Oncology (Chin.), vol. 20, no. 6, pp. 460-463, 2014.

[85] C. H. Xie, Z. Q. Wang, S. J. Guo, Z. X. Kang, P. Huang, and H. S. He, "Effect of Shenfu injection on bone marrow suppression and gastrointestinal symptoms in patients with non-small cell lung cancer chemotherapy," Jiangxi Medical Journal (Chin.), vol. 50, no. 8, pp. 795-797, 2015.

[86] Y. Q. Liu and J. W. Jia, "Shenmai injection combined with chemotherapy in the treatment of 30 cases of advanced nonsmall cell lung cancer," Clinical Cancer Research (Chin.), vol. 23, no. 8, pp. 557-558, 2011.

[87] Z. J. Yang, C. M. Deng, W. S. Mo, Y. H. Qiu, and F. Z. He, pp. 1-6, 2014, Effect of Shenmai injection combined with chemotherapy on quality of life in patients with advanced non-small cell lung cancer.//cancer professional committee of China society of integrated traditional Chinese and western medicine, China anti-cancer association. proceedings of the fifth international conference on traditional Chinese medicine, integrated traditional Chinese and western medicine oncology and the fourteenth national conference on integrated traditional Chinese and western medicine oncology.

[88] S. W. Luo, Y. P. Huang, H. L. Xia, Y. W. Yang, C. Mo, and $\mathrm{X}$. E. Wu, "Clinical observation of shenqi fuzheng injection combined with paclitaxel and cisplatin in the treatment of advanced non-small cell lung cancer," Chinese Clinical Oncology (Chin.), vol. 12, no. 5, pp. 381-382, 2007.

[89] F. L. Zhang, "The clinical observation of advanced non small cell lung cancer treated with shenqi fuzheng injection combined with PTX and DDP," Journal of Modern Oncology (Chin.), vol. 16, no. 7, pp. 1165-1166, 2008.

[90] L. Y. Wang, Q. S. Tu, J. H. Li, and H. J. Wu, "Clinical observation of shenqi fuzheng injection combined with chemotherapy in the treatment of advanced non-small cell lung cancer," Chinese Journal of Physics (Chin.), vol. 11, no. 10, pp. 1417-1418, 2009.

[91] Y. D. Chen, "Treatment of 48 cases of advanced lung cancer with non-small cell lung cancer by TP regimen combined with shenqi fuzheng injection," Chinese Journal of
Ethnomedicine and Ethnopharmacy (Chin.), vol. 19, no. 20, p. 95, 2010.

[92] H. Z. Cui, L. Zhu, Q. Niu, and J. Z. Guan, "Clinical observation of non-small cell lung cancer (NSCLC) treated with shenqi fuzheng injection combined with chemotherapy," Journal of Modern Oncology (Chin.), vol. 18, no. 5, pp. 925-926, 2010.

[93] H. Y. Mai, X. Y. Li, and J. W. Huang, "Observation of shenqi fuzheng injection: its effects on lessening the side effects of $\mathrm{TP}$ regime chemotherapy in patients with advanced non small-cell lung cancer," Guide China Medicine (Chin.), no. 23, pp. 423-425, 2013.

[94] D. H. Li and H. L. Yang, "Clinical observation of shenqi fuzheng injection combined with TP regimen in the treatment of advanced non-small cell lung cancer," Medicine Forum (Chin.), vol. 18, no. 10, pp. 1291-1292, 2014.

[95] T. Yuan, "Clinical significance of shenqi fuzheng injection on non-small cell lung cancer chemotherapy," Henan Medical Research (Chin.), vol. 23, no. 6, pp. 123-125, 2014.

[96] Y. Deng, Q. Ke, and Y. Mao, "Effect of shenqi fuzheng injection on hematopoietic and immune function in patients with advanced non-small cell lung cancer chemotherapy," Chinese Journal of Gerontology (Chin.), vol. 35, no. 21, pp. 6142-6144, 2015.

[97] X. L. Su, "Effect of shenqi fuzheng injection on immune function in patients with advanced non-small cell lung cancer after chemotherapy," World Latest Medicine Information (Electronic Version) (Chin.), vol. 16, no. 101, pp. 156-160, 2016.

[98] G. A. Xia, "Effect of Xiaoaiping injection combined with TP regimen in the treatment of advanced non-small cell lung cancer," Proceedings of Clinical Medicine (Chin.), vol. 22, no. 2, pp. 83-85, 2013.

[99] J. Yao, "Study on the efficacy and safety of Xiaoaiping injection combined with chemotherapy in advanced non-small cell lung cancer," Modern Journal of Integrated Traditional Chinese and Western Medicine (Chin.), vol. 25, no. 24, pp. 2706-2708, 2016.

[100] W. Y. Wang, Y. Zhou, X. J. Zhang, T. H. Gao, Z. F. Luo, and M. Y. Liu, "A random study of Xiaoaiping injection combined with chemotherapy on the treatment of advanced nonsmall cell lung cancer," Chinese Clinical Oncology (Chin.), vol. 14, no. 10, pp. 936-938, 2009.

[101] C. R. Mei, K. Wang, N. Lei, and Z. J. Mei, "Clinical observation of Xiaoaiping injection combined with TP regimen in the treatment of advanced non-small cell lung cancer," Journal of Chinese Pharmaceutical (Chin.), vol. 26, no. 11, pp. 1531-1533, 2015.

[102] D. E. Williams, P. C. Pairolero, C. S. Davis et al., "Survival of patients surgically treated for stage I lung cancer," The Journal of Thoracic and Cardiovascular Surgery, vol. 82, no. 1, pp. 70-76, 1981.

[103] G. M. Strauss, J. E. Herndon, M. A. Maddaus et al., “Adjuvant paclitaxel plus carboplatin compared with observation in stage IB non-small-cell lung cancer: CALGB 9633 with the cancer and leukemia group B, radiation therapy oncology group, and north central cancer treatment group study groups," Journal of Clinical Oncology, vol. 26, no. 31, pp. 5043-5051, 2015.

[104] M. W. Pan, Inhibitory Effect and Mechanism of Mesenchymal Stem Cells on NSCLC Cells, Beijing Jiaotong University, Beijing, China, 2018.

[105] D. R. Lu, D. P. Bai, S. Q. He et al., "Clinical study on aidi injection in zusanli (ST 36) in treating chemotherapy 
induced nausea and vomiting suffered from chemotherapy with cisplatin," World of Chinese Medicine (Chin.), vol. 13, no. 3, pp. 609-613, 2018.

[106] Y. Song and J. W. Gao, "Medical treatment of non-small cell lung cancer: an update," Journal of Postgraduate Medicine (Chin.), vol. 30, no. 11, pp. 1121-1127, 2017.

[107] S. Wang, "Research progress of kangai injection in the treatment of lung cancer," Clinical Cancer Research (Chin.), vol. 26, no. 2, pp. 136-139, 2014.

[108] Y. Q. Sun, L. Q. Wang, and L. P. Zhao, "Study on the antitumor effect of brucea javanica: chromatography-mass spectrometry analysis of 11-deesterified brucea oil," Journal of Shenyang Pharmaceutical University (Chin.), vol. 12, no. 14, pp. 13-23, 1981.

[109] D. Penzo, C. Tagliapietra, R. Colonna, V. Petronilli, and P. Bernardi, "Effects of fatty acids on mitochondria: implications for cell death," Biochimica et Biophysica Acta (BBA)Bioenergetics, vol. 1555, no. 1-3, pp. 160-165, 2002.

[110] Y. N. Zhang, L. Ma, and L. J. Wang, "Inhibitory effect of brucea javanica oil emulsion on gastric cancer cell proliferation and its mechanism," Journal of Practical Chinese with Modern Medicine (Chin.), vol. 16, pp. 282-283, 2003.

[111] X. W. Li, H. Wang, W. J. Qin, X. Li, T. Liu, and C. M. Fan, "Necrosis and apoptosis induced by brucea javanica oil emulsion in bladder cancer cell," Chinese Journal of Rehabilitation Theory and Practice (Chin.), vol. 10, pp. 163-164, 2004.

[112] L. Jiang, Y. M. Zhou, M. Chen, C. Zhou, and B. Li, "Early evaluation of the response of breast cancer to neoadjuvant chemotherapy with functional MRI," Chinese Journal of Breast Disease. (Electronic Version) (Chin.), vol. 5, no. 3, pp. 5-10, 2011.

[113] X. P. Shi and H. R. Zhao, "Research progress of brucea javanica oil," Northwest Pharmaceutical Journal (Chin.), vol. 25 , no. 3, p. 240, 2010.

[114] J. Pan and H. X. Bai, "Clinical value of combined detection of breast cancer tumor markers CA15-3 and CEA," Guide of China Medicine (Chin.), vol. 9, no. 26, pp. 265-266, 2011.

[115] C. X. Ding and Y. R. Suo, "Research progress on chemical constituents and pharmacology of traditional Chinese medicine brucea javanica," Traditional Chinese Patent Medicine (Chin.), vol. 28, no. 1, pp. 117-120, 2006.

[116] W. D. Zhou, L. Xiang, H. Q. Lu et al., "Radix codonopsi polysaccharide against 5-Fluorouracil-induced gastrointestinal mucositis in mice model," Liaoning Journal of Traditional Chinese Medicine (Chin.), vol. 43, no. 7, p. 1495, 2016.

[117] D. Zeng, Z. Y. Huang, D. K. Su, and Y. Y. Lu, "Effects of Codonopsis extract on reproductive performance and blood indexes of sow," Feed Research (Chin.), no. 13, p. 31, 2016.

[118] D. Y. Wang, B. Qu, and Z. P. Wang, "Clinical effects of the shenqi fuzheng injection on bone marrow suppression and immune function after small cell lung cancer chemotherapy," Clinical Journal of Chinese Medicine (Chin.), vol. 8, no. 11, p. 7, 2016. 